\title{
A MegaCam Survey of Outer Halo Satellites. I. Description of the Survey ${ }^{* \dagger}$
}

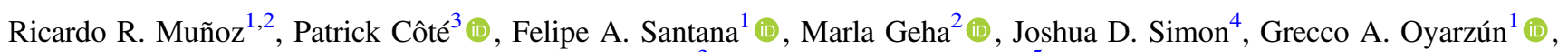 \\ Peter B. Stetson ${ }^{3}$ (D) and S. G. Djorgovski ${ }^{5}$ (i) \\ ${ }^{1}$ Departamento de Astronomía, Universidad de Chile, Camino del Observatorio 1515, Las Condes, Santiago, Chile; rmunoz@das.uchile.cl \\ ${ }^{2}$ Astronomy Department, Yale University, New Haven, CT 06520, USA \\ ${ }^{3}$ National Research Council of Canada, Herzberg Astronomy and Astrophysics, 5071 W. Saanich Road, Victoria, BC V9E 2E7, Canada \\ ${ }^{4}$ Observatories of the Carnegie Institution for Science, 813 Santa Barbara Street, Pasadena, CA 91101, USA \\ 5 Astronomy Department, California Institute of Technology, Pasadena, CA 91125, USA \\ Received 2017 August 10; revised 2018 March 20; accepted 2018 April 9; published 2018 June 12
}

\begin{abstract}
We describe a deep, systematic imaging study of satellites in the outer halo of the Milky Way. Our sample consists of 58 stellar overdensities-i.e., substructures classified as either globular clusters, classical dwarf galaxies, or ultra-faint dwarf galaxies-that are located at Galactocentric distances of $R_{\mathrm{GC}} \geqslant 25 \mathrm{kpc}$ (outer halo) and out to $\sim 400 \mathrm{kpc}$. This includes 44 objects for which we have acquired deep, wide-field, $g$ - and $r$-band imaging with the MegaCam mosaic cameras on the $3.6 \mathrm{~m}$ Canada-France-Hawaii Telescope and the $6.5 \mathrm{~m}$ Magellan-Clay telescope. These data are supplemented by archival imaging, or published $g r$ photometry, for an additional 14 objects, most of which were discovered recently in the Dark Energy Survey (DES). We describe the scientific motivation for our survey, including sample selection, observing strategy, data reduction pipeline, calibration procedures, and the depth and precision of the photometry. The typical $5 \sigma$ point-source limiting magnitudes for our MegaCam imaging-which collectively covers an area of $\approx 52 \mathrm{deg}^{2}$ - are $g_{\lim } \simeq 25.6$ and $r_{\text {lim }} \simeq 25.3 \mathrm{AB}$ mag. These limits are comparable to those from the coadded DES images and are roughly a half-magnitude deeper than will be reached in a single visit with the Large Synoptic Survey Telescope. Our photometric catalog thus provides the deepest and most uniform photometric database of Milky Way satellites available for the foreseeable future. In other papers in this series, we have used these data to explore the blue straggler populations in these objects, their density distributions, star formation histories, scaling relations, and possible foreground structures.
\end{abstract}

Key words: galaxies: dwarf - galaxies: fundamental parameters - galaxies: photometry - Galaxy: halo - globular clusters: general - Local Group

Supporting material: machine-readable table

\section{Introduction}

In the currently favored $\Lambda \mathrm{CDM}$ paradigm, the fossil record of galaxy formation is imprinted in the stellar halos that surround massive galaxies like the Milky Way. The number of substructures, or satellites, embedded in the halo is an important prediction of cosmological models-and one that depends sensitively on a number of parameters: the small-scale power spectrum, the assumed properties of the dark matter particles, and a variety of baryonic processes that can modify the shape of the satellite luminosity function. In principle, a comparison between the predicted and observed number of satellites can provide a straightforward test of cosmological models (Klypin et al. 1999; Moore et al. 1999). In practice, though, there are significant technical challenges and serious selection effects involved in finding and characterizing Galactic satellites.

Such satellites have historically been separated into two types of objects that are thought to have had dissimilar origins: globular clusters and dwarf galaxies. In recent years, it has become fashionable to further subdivide the latter category on the basis of luminosity or surface brightness: i.e., "classical" versus "ultra-faint" dwarf galaxies, although these are not

Based on observations obtained at the Canada-France-Hawaii Telescope (CFHT), which is operated by the National Research Council of Canada, the Institut National des Sciences de l'Univers of the Centre National de la Recherche Scientifique of France, and the University of Hawaii.

$\dagger$ This paper includes data gathered with the $6.5 \mathrm{~m}$ Magellan Telescopes located at Las Campanas Observatory, Chile. physically distinct classes. Beginning with the SDSS (York et al. 2000)-which provided uniform ugriz photometry covering a significant fraction of the sky-a large number of faint dwarf galaxies and globular clusters have been discovered. The number of known satellites has increased steadily for more than two centuries. Historically, sharp increases in the number of satellites have followed soon after the deployment of powerful new survey facilities (e.g., the telescopes of Herschel, the Oschin Schmidt, the SDSS, Pan-STARRS, and, most recently, the Dark Energy Camera (DECam) on the Blanco $4 \mathrm{~m}$ telescope).

As of early 2017, the Milky Way is known to contain at least 77 satellites located beyond a Galactocentric radius of $25 \mathrm{kpc}$. It is virtually certain that this number will continue to rise as observing facilities improve. In the next decade, the highly anticipated Large Synoptic Survey Telescope (LSST) should be especially important in improving our census of satellites, at least in the southern hemisphere.

In the meantime, it is desirable to characterize the properties of the known satellites in a careful and systematic way. Such efforts are presently hampered by the lack of uniform, highquality imaging for these objects, which are scattered over the entire sky and span wide ranges in apparent size, luminosity, and surface brightness. Existing catalogs containing photometric and structural parameters for the Milky Way satellites have tended to focus on either globular clusters and dwarf galaxies, despite the fact that the distinction between these stellar systems has become increasingly blurred over the years 
(i.e., at the lowest surface brightnesses, separating these populations is often impossible without spectroscopic information). Moreover, previous compilations continue to rely on shallow and heterogeneous data (with some of the early ones even photographic), some of it dating back to the $1960 \mathrm{~s}$ (see, e.g., Djorgovski 1993; Pryor \& Meylan 1993; Irwin \& Hatzidimitriou 1995; Trager et al. 1995; Harris 1996; Mateo 1998; McLaughlin \& van der Marel 2005; McConnachie 2012 and references therein).

Deep, homogeneous, digital photometry for a nearly complete sample of halo satellites would allow a fresh look into the nature of these satellites. Issues of particular interest include possible connections between the various sub-populations (globular clusters versus classical dwarfs versus ultrafaint dwarfs), including their density distributions (Plummer 1911; Hubble 1930; King 1962, 1966; Sérsic 1968; Wilson 1975; Elson et al. 1987), stellar populations, dark matter content, and evidence for tidal interactions with the Milky Way. Here, we introduce a deep, wide-field imaging survey of satellites belonging to the outer Galactic halo. The survey, which makes no a priori selection on the basis of object morphology or classification, is based on homogeneous $g$ - and $r$-band imaging for 44 objects obtained with mosaic CCD cameras on the $3.6 \mathrm{~m}$ Canada-Hawaii-France Telescope (CFHT) and the $6.5 \mathrm{~m}$ Magellan/Clay telescope. Our imaging is supplemented with photometry assembled from the literature - or derived from our reduction and analysis of archival imaging-for an additional 14 objects. In 2015, when the analysis of our secondary targets was completed, the survey provided uniform photometry for a nearly complete ( $\gtrsim 95 \%)$ sample of 60 satellites located more than $25 \mathrm{kpc}$ from the Galactic center. Since that time, a number of additional satellites have been detected, primarily by the Dark Energy Survey (DES), so our sample now represents roughly threequarters of the 81 cataloged members of the outer halo.

Our survey is the first to combine depth, wide areal coverage, multi-band imaging, and a high level of completeness for outer halo satellites. In this paper, we describe the survey strategy, sample selection, data reduction, and calibration. Previous papers in this series have examined the internal dynamics of the unusual stellar system Palomar 13 (Bradford et al. 2011), reported the discovery of Munoz 1, an extremely low-luminosity star cluster in the field of the Ursa Minor dwarf galaxy (Muñoz et al. 2012a), studied the properties of blue straggler stars in remote satellites (Santana et al. 2013), examined possible foreground populations in the direction of NGC 2419 and Koposov 2 (Carballo-Bello et al. 2015), and characterized the Sagittarius tidal stream in the vicinity of the globular cluster Whiting 1 (Carballo-Bello et al. 2017). A companion paper (Muñoz et al. 2018) presents homogeneously derived structural parameters and future papers will explore the density distributions of our sample objects and compare their photometric and structural parameters to those of other stellar systems.

This paper is organized as follows. In Section 2, we discuss the sample selection for our survey. In Section 3, we describe the observing strategy and data reduction procedures for our primary sample of 44 objects observed with the CFHT or Clay telescopes. In Section 4, we discuss the assembly of published or archival data for our secondary sample of 14 recently discovered objects that were not included in the primary sample. Section 5 discusses some consistency checks on our photometry, while Section 6 presents some illustrative results from our program. We present a summary and conclusion in Section 7.

\section{Selection of Targets}

The goal of our survey is a homogeneous study of the photometric and structural parameters for a large, unbiased sample of satellites residing in the outer halo of the Milky Way. This is a significant undertaking, as it requires deep, uniform, wide-field, and multi-filter imaging for dozens of objects that span a wide range in luminosity, surface brightness and distance, and are scattered across the northern and southern skies. Initially, our survey was designed to rely entirely on CFHT and Magellan imaging for 41 Galactic satellites-a complete list of Galactic satellites as of 2009 (excluding Sagittarius and the Magellanic Clouds). Henceforth, we shall refer to this sample-and three more objects that were discovered after the start of the survey-as our "primary sample" (see Section 2.1). In Section 2.2, we discuss a "secondary sample" of 14 satellites discovered in 2013, 2014, or 2015 that relies on published or archival data.

\subsection{Primary Sample}

In an important departure from most previous studies, we make no a priori selection on satellite morphology or classification. This seems prudent since, as discussed in Section 1, the once-clear distinction between globular clusters, classical dwarf galaxies, and ultra-faint dwarf galaxies has become increasingly blurred in recent years. Rather, our sample is defined purely on the basis of location in the Galactic halo, with all overdensities located beyond a Galactocentric distance of $R_{\mathrm{GC}}=25 \mathrm{kpc}$ considered appropriate targets. Although somewhat arbitrary, this choice for the inner boundary of the "outer halo" seems reasonable given that inside this radius, halo stars tend to have higher metallicities and different kinematics than their more distance counterparts (see, e.g., Carollo et al. 2007, 2010 and references therein). While we impose no firm cutoff on outer radius, we do confine ourselves to satellites that are believed to be members of the Milky Way satellite system (e.g., Mateo 1998; McConnachie 2012). Our most distant satellite, Leo T, lies at $D_{\odot} \approx 417 \mathrm{kpc}$-a point at which the distinction between membership in the Galactic and Local Group satellite subsystems becomes unclear. Our next most distant objects-Leo I and Leo II-lie at distances of 254 and $233 \mathrm{kpc}$, respectively. Thus, our survey focuses on the population of satellites between $R_{\mathrm{GC}}=25 \mathrm{kpc}$ and the virial radius of the Milky Way, which various estimates place between 200 and $300 \mathrm{kpc}$ (e.g., Klypin et al. 2002; Dehnen et al. 2006; Xue et al. 2008).

Based on the above criteria, we identified in 2009 a total of 41 satellites belonging to the outer halo of the Milky Way. This sample includes a mixture of globular clusters (Harris 1996, 2010) and classical or ultra-faint dwarf galaxies (see, e.g., the compendia of Mateo 1998 and McConnachie 2012). Three massive satellites-the LMC, SMC, and Sagittarius dwarf spheroidal galaxy-were deemed too large and luminous to observe efficiently within the context of this survey and were excluded. At the same time, two satellites that were discovered after our survey began-Segue 3 and PisceS II (Belokurov et al. 2010)—were added to our sample during the 2010A semester. A third object residing in the outer 
halo- the ultra-faint globular cluster Muñoz 1, which is offset by $\sim 1.8$ from the center of the Ursa Minor dwarf galaxy but located well in the foreground-was, in fact, discovered serendipitously in this survey (Muñoz et al. 2012b). Thus, our primary sample consists of 44 objects that, aside from the aforementioned cases of the LMC, SMC, and Sagittarius, represents a complete sample of outer halo satellites as of 2010.

Table 1 gives some basic information for these 44 objects. From left to right, the columns record ID number, adopted name, other names appearing in the literature, position in equatorial $(\alpha, \delta)$ and Galactic $(l, b)$ coordinates, reddening $E(B-V)$ from Schlafly \& Finkbeiner (2011), Galactocentric distance and Cartesian coordinates in a Galactocentric frame,

$$
\begin{aligned}
X & =R_{\odot} \cos b \cos l-X_{\odot} \\
Y & =R_{\odot} \cos b \sin l \\
Z & =R_{\odot} \sin b,
\end{aligned}
$$

where $X_{\odot}=8.5 \mathrm{kpc}$ is our adopted distance to the Galactic center, and $R_{\odot}$ is the heliocentric distance to each object, so $R_{\mathrm{GC}}=\sqrt{X^{2}+Y^{2}+Z^{2}}$ is the distance to the Galactic center. For each satellite, references to the original discovery paper or papers are given in the final column.

As described in Section 2.2, this sample has been supplemented by an additional 14 satellites discovered between mid2010, when data acquisition for our primary sample was completed, and 2015. This brings our total sample to 58 objects, which, in 2015, represented $\simeq 95 \%$ of all known Milky Way satellites beyond $25 \mathrm{kpc}$ (i.e., excluding the three massive systems).

Figure 1 shows three projections $(Y Z, X Z$, and $X Y)$ of our sample in Cartesian coordinates centered on the Milky Way center (Equation (1)). Objects belonging to our primary and secondary samples are shown as red and blue crosses, respectively. Globular clusters at $R_{\mathrm{GC}}<25 \mathrm{kpc}$ are denoted by gray squares, and satellites absent from our study are shown as green crosses. The inner and outer circles plotted in each panel show our adopted boundary for the outer halo and the virial radius of the Milky Way according to Dehnen et al. (2006), respectively. The arrow indicates the direction to M31 in each projection. Figure 2 shows the distribution of these same populations on the sky, in equatorial and Galactic coordinates (upper and lower panel, respectively).

\subsection{Secondary Sample}

Data acquisition for the 44 objects belonging to our primary sample was completed in mid-2011. Between that time and 2015, a number of new Galactic satellites were identified, many located beyond the Galactocentric distance of $R_{\mathrm{GC}}=25 \mathrm{kpc}$ that defines the boundary of the outer halo sample. We therefore define a "secondary" sample for our survey that is listed in Table 2. This table presents the same basic information for the 14 new Milky Way satellites as Table 1 did for our primary sample. From left to right, the columns of this table give ID number, adopted name, other names appearing in the literature, position in equatorial and Galactic coordinates, reddening from Schlafly \& Finkbeiner (2011), Galactocentric distance, Cartesian coordinates in a Galactocentric frame (Equation (1)), and references to the original discovery paper or papers.

The 14 objects in our secondary sample include a mixture of probable or confirmed dwarf galaxies, probable or confirmed globular clusters, and objects whose classification remains ambiguous at the present time (i.e., dynamical masses and metallicity distribution functions are needed to surmise their true nature). In every case, the initial discovery was based on survey data acquired with wide-field imaging telescopes. For instance, imaging from Pan-STARRS led to the detection of Triangulum I I (Laevens et al. 2015b) while Crater was discovered independently by Laevens et al. (2014) using PanSTARRS and by Belokurov et al. (2014) using VST/ATLAS. ${ }^{6}$ Three additional satellites were discovered in SDSS imaging: Balbinot 1 (Balbinot et al. 2013), Kim 1 (Kim \& Jerjen 2015a), and Pegasus 3 (Kim et al. 2015a). However, it has been the deployment of the DECam (Flaugher et al. 2015) on the Blanco telescope that has produced the largest harvest of satellites. Seven total faint stellar systems-Eridanus 3, Horologium I, Reticulum II, Eridanus II, Pictoris I, Tucana 2, and Phoenix 2-were identified by two independent groups (Koposov et al. 2015a; The DES Collaboration et al. 2015) using imaging from the Dark Energy Survey (DES; Diehl et al. 2014). An eighth DES satellite, Grus I, was discovered by Koposov et al. (2015a), and a ninth, Indus 1, was actually discovered by Kim et al. (2015b) using DECam imaging obtained as part of the Stromlo Milky Way Satellite Survey and later identified in the DES (Koposov et al. 2015a; The DES Collaboration et al. 2015). A tenth object, Horologium II, was identified by Kim \& Jerjen (2015b) using DES Y1A1 public data, while Hydra I I was discovered by Martin et al. (2015) in their DECam Survey of the MAgellanic Stellar History (SMASH).

At this stage, the nature of many of these satellites is an area of active investigation. Spectroscopy for member stars in a handful of systems has provided velocity dispersions, mass-tolight ratios, and elemental abundances for a few objects, allowing them to be classified as either dwarf galaxies (e.g., Horologium I, Reticulum II, Hydra II; Kirby et al. 2015; Koposov et al. 2015b; Simon et al. 2015) or globular clusters (Crater; Kirby et al. 2015). But for several of the new satellites, only preliminary classifications are availableusually based on their structural properties.

In this survey, we are most concerned with the measurement of structural properties from homogeneous, high-quality CCD imaging. Fortuitously, for nearly all of these objects, either the discovery or follow-up observations include imaging in the SDSS $g$ and $r$ filters - the same filter combination used for our primary survey. It is therefore possible to maintain the uniformity and homogeneity of the primary survey by adding published photometry, or photometry derived from data in the archive, for these newly discovered satellites. Details on the photometric catalogs for our secondary objects-including both photometry assembled from the literature and photometry obtained using data retrieved from public archives-will be presented in Section 4.

It is worth noting that the number of known Galactic satellites continues to rise, with many objects identified since 2015 (e.g., Kim \& Jerjen 2015b; Kim et al. 2015a; Koposov et al. 2015a; Laevens et al. 2015a, 2015b; The DES Collaboration et al. 2015; Homma et al. 2016; Luque et al. 2016, 2017; Torrealba et al. 2016a, 2016b). At the time

\footnotetext{
6 A French amateur astronomer, Pascal Le Dû, also discovered Crater using a small aperture telescope. He published his results in the French magazine L'Astronomie in 2014 January. The article can be found at http://www. cielocean.fr/uploads/images/FichiersPDF/L-Astronomie-_Janvier2014.pdf.
} 
Table 1

Basic Parameters for Outer Halo Objects: Primary Sample

\begin{tabular}{|c|c|c|c|c|c|c|c|c|c|c|c|c|c|}
\hline No. & Name & Other & $\begin{array}{r}\alpha(2000) \\
(\operatorname{deg})\end{array}$ & $\begin{array}{r}\delta(2000) \\
(\mathrm{deg})\end{array}$ & $\begin{array}{r}l \\
(\mathrm{deg})\end{array}$ & $\begin{array}{r}b \\
(\operatorname{deg})\end{array}$ & $\begin{array}{c}E(B-V) \\
(\mathrm{mag})\end{array}$ & $\begin{array}{r}R_{\odot} \\
(\mathrm{kpc})\end{array}$ & $\begin{array}{r}R_{\mathrm{GC}} \\
(\mathrm{kpc})\end{array}$ & $\begin{array}{r}X \\
(\mathrm{kpc})\end{array}$ & $\begin{array}{r}Y \\
(\mathrm{kpc})\end{array}$ & $\begin{array}{r}Z \\
(\mathrm{kpc})\end{array}$ & $\begin{array}{l}\text { Discovery } \\
\text { References }\end{array}$ \\
\hline 1 & Sculptor & $\ldots$ & 15.0183 & -33.7186 & 287.6964 & -83.1523 & 0.016 & 86.0 & 86.1 & -5.4 & -9.8 & -85.4 & 1 \\
\hline 2 & Whiting 1 & $\ldots$ & 30.7372 & -3.2519 & 161.6161 & -60.6353 & 0.021 & 30.1 & 34.9 & -22.4 & 4.7 & -26.3 & 2 \\
\hline 3 & Segue 2 & $\ldots$ & 34.8226 & +20.1625 & 149.4461 & -38.1444 & 0.164 & 35.0 & 41.2 & -32.2 & 14.0 & -21.6 & 3 \\
\hline 4 & Fornax & $\ldots$ & 39.9583 & -34.4997 & 237.2382 & -65.6740 & 0.020 & 147.0 & 149.1 & -41.4 & -50.9 & -133.9 & 4 \\
\hline 5 & AM 1 & E 1 & 58.7607 & -49.6153 & 258.3610 & -48.4700 & 0.007 & 123.3 & 124.7 & -25.0 & -80.1 & -92.3 & 5 \\
\hline 6 & Eridanus & $\ldots$ & 66.1853 & -21.1876 & 218.1069 & -41.3325 & 0.018 & 90.1 & 95.4 & -61.7 & -41.8 & -59.5 & 6 \\
\hline 7 & Palomar 2 & $\ldots$ & 71.5248 & +31.3817 & 170.5302 & -9.0719 & 1.015 & 27.2 & 35.5 & -35.0 & 4.4 & -4.3 & 7,8 \\
\hline 8 & Carina & $\ldots$ & 100.4066 & -50.9593 & 260.1061 & -22.2194 & 0.053 & 105.0 & 106.7 & -25.2 & -95.8 & -39.7 & 9 \\
\hline 9 & NGC 2419 & $\ldots$ & 114.5354 & +38.8819 & 180.3698 & +25.2416 & 0.052 & 82.6 & 90.4 & -83.2 & -0.5 & 35.2 & 10 \\
\hline 10 & Koposov 2 & $\ldots$ & 119.5715 & +26.2574 & 195.1098 & +25.5469 & 0.037 & 34.7 & 42.3 & -38.7 & -8.2 & 15.0 & 11 \\
\hline 11 & UMa II & $\ldots$ & 132.8726 & +63.1335 & 152.4603 & +37.4411 & 0.082 & 32.0 & 38.5 & -31.0 & 11.8 & 19.4 & 12 \\
\hline 12 & Pyxis & $\ldots$ & 136.9869 & -37.2266 & 261.3212 & +6.9915 & 0.282 & 39.4 & 41.5 & -14.4 & -38.7 & 4.8 & 13 \\
\hline 13 & Leo $\mathrm{T}$ & $\ldots$ & 143.7292 & +17.0482 & 214.8598 & +43.6657 & 0.027 & 417.0 & 422.1 & -256.0 & -172.4 & 287.9 & 14 \\
\hline 14 & Palomar 3 & UGC 05439 & 151.3823 & +0.0718 & 240.1409 & +41.8642 & 0.037 & 92.5 & 96.0 & -42.8 & -59.8 & 61.7 & 7,8 \\
\hline 15 & Segue 1 & $\ldots$ & 151.7504 & +16.0756 & 220.4777 & +50.4089 & 0.028 & 23.0 & 28.1 & -19.6 & -9.5 & 17.7 & 15 \\
\hline 16 & Leo I & UGC 5470, DDO 74 & 152.1146 & +12.3059 & 225.9848 & +49.1100 & 0.031 & 254.0 & 258.0 & -124.0 & -119.6 & 192.0 & 16 \\
\hline 17 & Sextans & $\ldots$ & 153.2628 & -1.6133 & 243.4974 & +42.2736 & 0.041 & 86.0 & 89.2 & -36.9 & -57.0 & 57.9 & 17 \\
\hline 18 & UMa I & $\ldots$ & 158.7706 & +51.9479 & 159.3625 & +54.4269 & 0.016 & 97.0 & 101.9 & -61.3 & 19.9 & 78.9 & 18 \\
\hline 19 & Willman I & SDSS J1049 + 5103 & 162.3436 & +51.0501 & 158.5730 & +56.7834 & 0.012 & 38.0 & 43.0 & -27.9 & 7.6 & 31.8 & 19 \\
\hline 20 & Leo II & $\begin{array}{l}\text { Leo B, UGC } 6253 \\
\text { DDO } 93\end{array}$ & 168.3627 & +22.1529 & 220.1611 & +67.2251 & 0.014 & 233.0 & 235.7 & -77.4 & -58.2 & 214.8 & 16 \\
\hline 21 & Palomar 4 & UGCA 237 & 172.3180 & +28.9733 & 202.3122 & +71.8009 & 0.020 & 108.7 & 111.5 & -39.9 & -12.9 & 103.3 & 7,8 \\
\hline 22 & Leo V & $\ldots$ & 172.7857 & +2.2194 & 261.8565 & +58.5343 & 0.024 & 178.0 & 178.8 & -21.7 & -92.0 & 151.8 & 20 \\
\hline 23 & Leo IV & $\ldots$ & 173.2405 & -0.5453 & 265.4577 & +56.5059 & 0.022 & 154.0 & 154.6 & -15.2 & -84.7 & 128.4 & 15 \\
\hline 24 & Koposov 1 & $\ldots$ & 179.8253 & +12.2615 & 260.9700 & +70.7551 & 0.022 & 48.3 & 49.5 & -11.0 & -15.7 & 45.6 & 11 \\
\hline 25 & ComBer & $\ldots$ & 186.7454 & +23.9069 & 241.8645 & +83.6122 & 0.015 & 44.0 & 45.2 & -10.8 & -4.3 & 43.7 & 15 \\
\hline 26 & CVn II & SDSS J1257 + 3419 & 194.2927 & +34.3226 & 113.5747 & +82.7013 & 0.009 & 160.0 & 160.7 & -16.6 & 18.6 & 158.7 & 15,21 \\
\hline 27 & CVn I & $\ldots$ & 202.0091 & +33.5521 & 74.3039 & +79.8289 & 0.012 & 218.0 & 217.8 & 1.9 & 37.1 & 214.6 & 22 \\
\hline 28 & AM 4 & $\ldots$ & 209.0883 & -27.1635 & 320.2827 & +33.5116 & 0.046 & 32.2 & 27.5 & 12.1 & -17.2 & 17.8 & 23 \\
\hline 29 & Bootes II & $\ldots$ & 209.5141 & +12.8553 & 353.7311 & +68.8649 & 0.026 & 42.0 & 39.8 & 6.6 & -1.6 & 39.2 & 24 \\
\hline 30 & Bootes I & $\ldots$ & 210.0200 & +14.5135 & 358.1017 & +69.6366 & 0.015 & 66.0 & 63.5 & 14.4 & -0.8 & 61.9 & 25 \\
\hline 31 & NGC 5694 & $\ldots$ & 219.9019 & -26.5391 & 331.0560 & +30.3594 & 0.086 & 35.0 & 29.1 & 17.9 & -14.6 & 17.7 & 26 \\
\hline 32 & Muñoz 1 & $\ldots$ & 225.4490 & +66.9682 & 105.4414 & +45.4808 & 0.021 & 45.0 & 47.3 & -16.9 & 30.4 & 32.1 & 27 \\
\hline 33 & NGC 5824 & $\ldots$ & 225.9943 & -33.0685 & 332.5548 & +22.0702 & 0.148 & 32.1 & 25.6 & 17.9 & -13.7 & 12.1 & 28 \\
\hline 34 & Ursa Minor & UGC 9749, DDO 199 & 227.24 & +67.2221 & 104.9818 & +44.8127 & 0.028 & 76.0 & 78.0 & -22.4 & 52.1 & 53.6 & 8 \\
\hline 35 & Palomar 14 & AvdB & 242.7544 & +14.9584 & 28.7472 & +42.1902 & 0.030 & 76.5 & 71.3 & 41.2 & 27.2 & 51.4 & 29 \\
\hline 36 & Hercules & $\ldots$ & 247.7722 & +12.7852 & 28.7275 & +36.8564 & 0.053 & 132.0 & 126.2 & 84.1 & 50.8 & 79.2 & 15 \\
\hline 37 & NGC 6229 & $\ldots$ & 251.7454 & +47.5276 & 73.6383 & +40.3064 & 0.020 & 30.5 & 29.9 & -2.0 & 22.3 & 19.7 & 30 \\
\hline 38 & Palomar 15 & UGC 10642 & 254.9626 & -0.5390 & 18.8485 & +24.3370 & 0.338 & 45.1 & 38.0 & 30.4 & 13.3 & 18.6 & 31 \\
\hline 39 & Draco & UGC 10822, DDO 208 & 260.0684 & +57.9185 & 86.3710 & +34.7128 & 0.024 & 76.0 & 76.0 & -4.5 & 62.4 & 43.3 & 8 \\
\hline 40 & NGC 7006 & $\ldots$ & 315.3722 & +16.1871 & 63.7691 & -19.4068 & 0.071 & 41.2 & 38.4 & 8.7 & 34.9 & -13.7 & 26 \\
\hline 41 & Segue 3 & $\ldots$ & 320.3795 & +19.1178 & 69.3998 & -21.2723 & 0.084 & 27.0 & 25.5 & 0.3 & 23.6 & -9.8 & 32 \\
\hline 42 & Pisces II & $\ldots$ & 344.6345 & +5.9526 & 79.2125 & -47.1089 & 0.056 & 182.0 & 181.1 & 14.7 & 121.7 & -133.3 & 32 \\
\hline 43 & Palomar 13 & UGCA 435 & 346.6858 & +12.7712 & 87.1038 & -42.7007 & 0.098 & 26.0 & 27.1 & -7.5 & 19.1 & -17.6 & 7,8 \\
\hline 44 & NGC 7492 & $\ldots$ & 347.1102 & -15.6108 & 53.3865 & -63.4764 & 0.031 & 26.3 & 25.4 & -1.5 & 9.4 & -23.5 & 30 \\
\hline
\end{tabular}

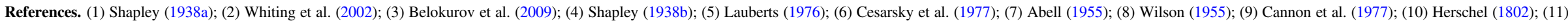

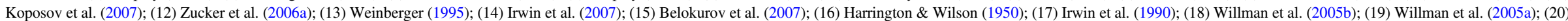

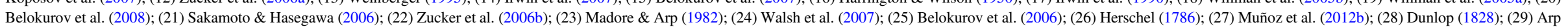
$\&$ van den Bergh (1960); (30) Herschel (1789); (31) Zwicky (1959); (32) Belokurov et al. (2010). 


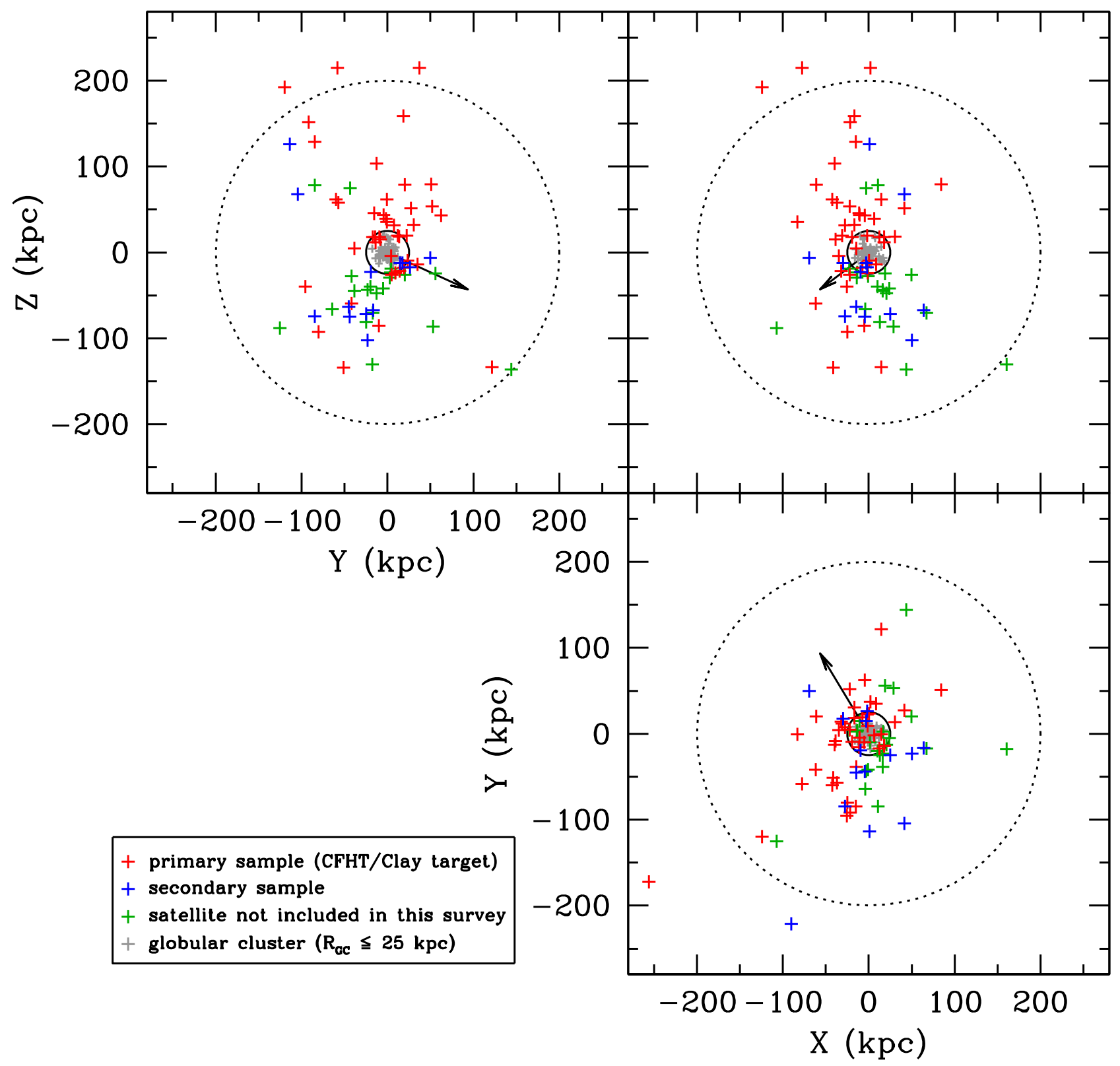

Figure 1. Distribution of Milky Way satellites in a Cartesian coordinate system centered on the Galactic center. The 44 objects in our primary sample (i.e., having CFHT or Clay imaging) are indicated by the red crosses. The blue crosses indicate objects in our expanded sample: i.e., recently discovered satellites whose structural parameters are examined using archival or literature data. The gray crosses show Galactic globular clusters, while the green crosses indicate known satellites, as of 2017, that are absent from our study. The inner circle at $25 \mathrm{kpc}$ indicates the Galactocentric radius that defines the boundary of study of "outer halo" substructures. The dashed circle at $200 \mathrm{kpc}$ shows the virial radius of the Milky Way (e.g., Dehnen et al. 2006).

of writing, our sample represents roughly three-quarters of the 81 known satellites having $R_{\mathrm{GC}} \geqslant 25 \mathrm{kpc}$. Muñoz et al. (2018) gives more details on those satellites that are absent from our primary and secondary samples.

\section{Imaging and Data Reductions for Primary Targets}

For our primary sample, observations for northern hemisphere objects were carried out using the MegaCam instrument on the $3.6 \mathrm{~m} \mathrm{CFHT}$. In the south, observations were made using the mosaic camera-also named Megacam-on the $6.5 \mathrm{~m}$ Magellan II-Clay telescope. To avoid confusion we henceforth refer to these instruments as CFHT-MegaCam and
Clay-Megacam. Table 3 summarizes the details of the six CFHT and Clay observing programs, amounting to $\sim 100 \mathrm{hr}$ of telescope time, that comprise our survey.

\subsection{CFHT-MegaCam Imaging}

CFHT-MegaCam is a wide-field imager consisting of 36 CCDs-each measuring $2048 \times 4612$ pixels-that together cover a $0.96 \times 0.94$ field of view at a scale of 0 !' 187 pixel $^{-1}$ (Boulade et al. 2003). All observations were carried out in queue mode during the 2009-A, 2009-B, and 2010-A observing semesters. Table 4 provides details on the observations for our primary objects. From left to right, the columns of this table 

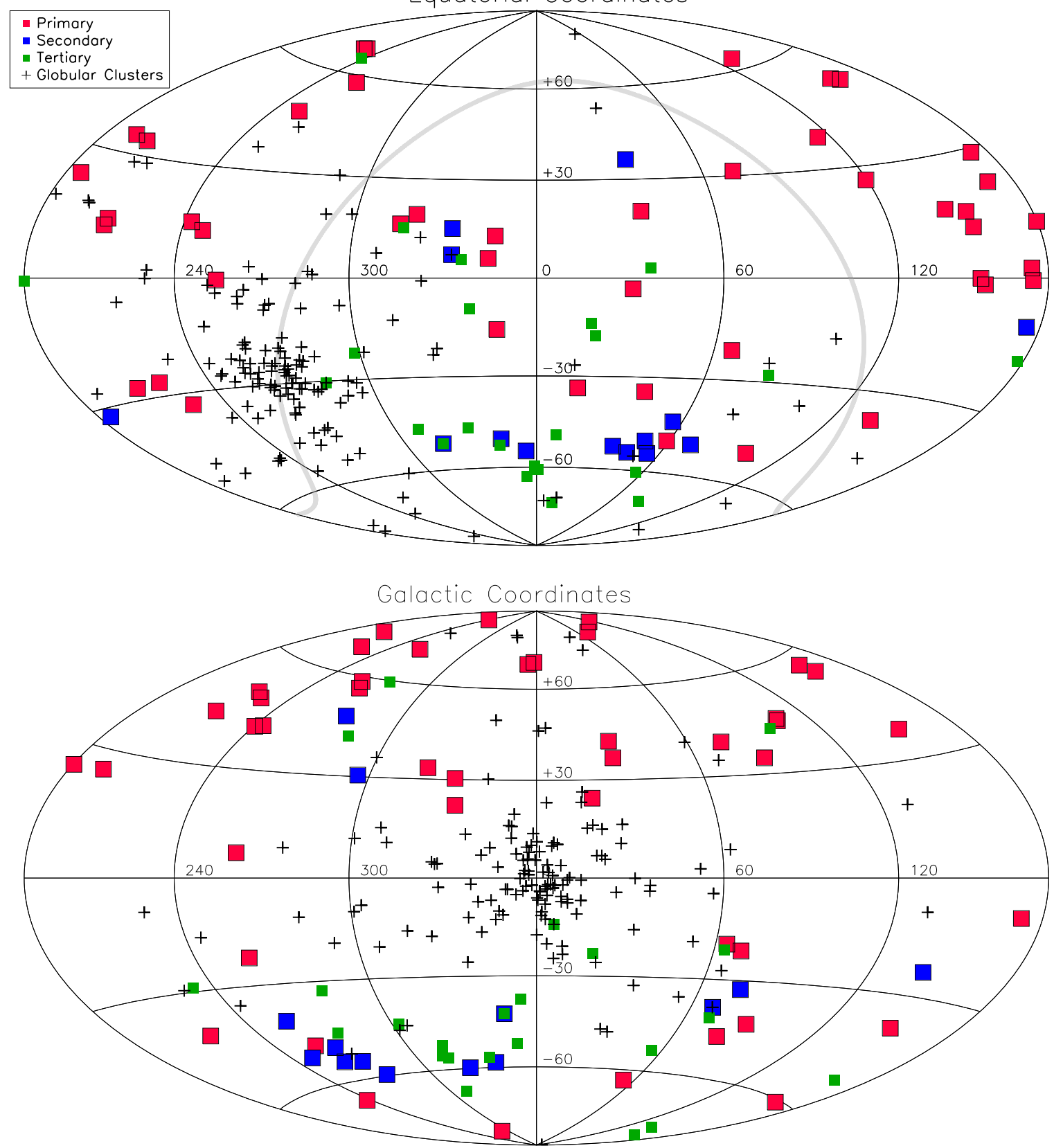

Figure 2. Upper panel: Aitoff projection in equatorial coordinates of the distribution of our program objects on the sky. The red squares show objects with CFHT or Clay imaging. The blue squares indicate objects in our expanded (secondary) sample with structural parameters derived from archival or literature data. Galactic globular clusters with $R_{\mathrm{GC}}<25 \mathrm{kpc}$ are shown as gray squares. Galaxies that were not included in our study are shown as green squares. Lower panel: same as the upper panel except in Galactic coordinates.

record the target name, telescope, mosaic geometry, total areal coverage, source of astrometric and photometric calibration (see Sections 3.3 and 3.4, respectively), mean airmass, and total exposure time in the $g$ and $r$ bandpasses. Although the color baseline offered by these two filters is limited, their choice allows us to minimize exposure times for color-magnitude diagram (CMD) analyses (see Section 5.2). Exposure times were chosen so that the $5 \sigma$ point-source limiting magnitude for all objects, and in both bands, lie $\sim 2-3$ magnitudes below the main-sequence turnoff (MSTO).

For 22 of our 30 CFHT targets, a single pointing was adequate to provide complete coverage. For the remaining eight objects, a grid of either $2 \times 1$ or $2 \times 2$ pointings was used, depending on the spatial extent of the satellite. In all cases, a series of dithered exposures was collected, usually in dark conditions. The dithering pattern used was selected from the standard CFHT-MegaCam operation options to provide coverage of both the small and large gaps between chips (i.e., the largest vertical gaps in MegaCam are six times wider than the small gaps). Typical image quality for the CFHT imaging is $\approx 0$ !'7-0!" 9 . Altogether, the CFHT component of our MegaCam survey covers a total area of $43.25 \mathrm{deg}^{2}$.

For two program objects-Pal 3 and NGC 7492-imaging was collected using both facilities as a cross-check on our photometry and astrometry. The results of this comparison will be presented in Section 5.1 below. 
Table 2

Basic Parameters for Outer Halo Objects: Secondary Sample

\begin{tabular}{|c|c|c|c|c|c|c|c|c|c|c|c|c|c|}
\hline No. & Name & Other & $\begin{array}{r}\alpha(2000) \\
(\mathrm{deg})\end{array}$ & $\begin{array}{r}\delta(2000) \\
(\mathrm{deg})\end{array}$ & $\begin{array}{r}l \\
(\mathrm{deg})\end{array}$ & $\begin{array}{r}b \\
(\operatorname{deg})\end{array}$ & $\begin{array}{c}E(B-V) \\
(\mathrm{mag})\end{array}$ & $\begin{array}{r}R_{\odot} \\
(\mathrm{kpc})\end{array}$ & $\begin{array}{r}R_{\mathrm{GC}} \\
(\mathrm{kpc})\end{array}$ & $\begin{array}{r}X \\
(\mathrm{kpc})\end{array}$ & $\begin{array}{r}Y \\
(\mathrm{kpc})\end{array}$ & $\begin{array}{r}Z \\
(\mathrm{kpc})\end{array}$ & References \\
\hline 1 & Triangulum II & Laevens 2 & 33.3252 & +36.1702 & 140.9044 & -23.8281 & 0.068 & 30.0 & 36.5 & -29.8 & 17.3 & -12.1 & 1 \\
\hline 2 & Eridanus 3 & $\ldots$ & 35.6952 & -52.2838 & 274.9547 & -59.5966 & 0.022 & 87.0 & 87.0 & -4.6 & -43.9 & -75.0 & 2,3 \\
\hline 3 & Horologium I & $\ldots$ & 43.8812 & -54.1160 & 271.3843 & -54.7350 & 0.013 & 79.0 & 79.3 & -7.3 & -45.6 & -64.5 & 2,3 \\
\hline 4 & Horologium II & $\ldots$ & 49.1076 & -50.0486 & 262.5314 & -54.1391 & 0.018 & 78.0 & 79.1 & -14.3 & -45.3 & -63.2 & 4 \\
\hline 5 & Reticulum II & $\ldots$ & 53.9203 & -54.0513 & 266.3007 & -49.7376 & 0.016 & 30.0 & 31.5 & -9.7 & -19.4 & -22.9 & 2,3 \\
\hline 6 & Eridanus II & $\ldots$ & 56.0925 & -43.5329 & 249.7802 & -51.6431 & 0.008 & 380.0 & 381.9 & -89.7 & -221.4 & -298.0 & 2,3 \\
\hline 7 & Pictoris I & $\ldots$ & 70.9490 & -50.2854 & 257.3020 & -40.6438 & 0.011 & 114.0 & 115.7 & -27.4 & -84.4 & -74.3 & 2,3 \\
\hline 8 & Crater & $\begin{array}{c}\text { Laevens 1 } \\
\text { PSO J174.0675-10.8774 }\end{array}$ & 174.0668 & -10.8772 & 274.8070 & +47.8473 & 0.023 & 170.0 & 169.9 & 1.2 & -113.7 & 126.0 & 5,6 \\
\hline 9 & Hydra II & $\cdots$ & 185.4251 & -31.9860 & 295.6171 & +30.4630 & 0.052 & 134.0 & 131.1 & 41.6 & -104.1 & 67.9 & 7 \\
\hline 10 & Indus 1 & Kim 2 & 317.2020 & -51.1671 & 347.1550 & -42.0692 & 0.026 & 100.0 & 94.0 & 63.9 & -16.4 & -67.0 & $8,2,3$ \\
\hline 11 & Balbinot 1 & $\cdots$ & 332.6791 & +14.9403 & 75.1723 & -32.6441 & 0.051 & 31.9 & 31.2 & -1.7 & 26.0 & -17.2 & 9 \\
\hline 12 & Kim 1 & $\ldots$ & 332.9214 & +7.0271 & 68.5158 & -38.4240 & 0.070 & 19.8 & 19.2 & -2.8 & 14.4 & -12.3 & 10 \\
\hline 13 & Grus I & $\ldots$ & 344.1798 & -50.1800 & 338.6512 & -58.2367 & 0.007 & 120.0 & 116.1 & 50.4 & -22.9 & -102.0 & 3 \\
\hline 14 & Phoenix 2 & $\cdots$ & 354.9960 & -54.4115 & 323.6820 & -59.7438 & 0.012 & 83.0 & 79.9 & 25.2 & -24.7 & -71.7 & 2,3 \\
\hline
\end{tabular}

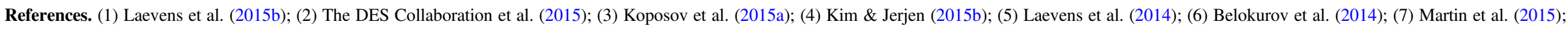
(8) Kim et al. (2015b); (9) Balbinot et al. (2013); (10) Kim \& Jerjen (2015a); (11) Kim et al. (2015a). 
Table 3

Observing Run Information

\begin{tabular}{|c|c|c|c|c|c|c|c|c|c|}
\hline Telescope & Instrument & PI & $N_{\mathrm{CCD}}$ & $\begin{array}{l}\text { Field } \\
(\text { deg) }\end{array}$ & $\begin{array}{c}\text { Scale } \\
(" / \text { pixel })\end{array}$ & $\begin{array}{c}\text { FWHM } \\
\left({ }^{\prime \prime}\right)\end{array}$ & Filters & ID \# & Date \\
\hline CFHT & MegaCam & Côté & 36 & $0.96 \times 0.94$ & 0.187 & $0.7-0.9$ & $g, r$ & $\begin{array}{l}\text { 09AC07 } \\
09 \mathrm{BC} 02 \\
10 \mathrm{AC} 06\end{array}$ & $\begin{array}{l}2009-A \\
2009-B \\
2010-A\end{array}$ \\
\hline Clay & MegaCam & $\begin{array}{l}\text { Geha } \\
\text { Simon } \\
\text { Simon }\end{array}$ & 36 & $0.40 \times 0.40$ & 0.160 & $0.7-1.1$ & $g, r$ & $\begin{array}{c}\text { 2010B-0472 } \\
\ldots \\
\ldots\end{array}$ & $\begin{array}{l}2010-B \\
2010-B \\
2011-A\end{array}$ \\
\hline
\end{tabular}

Table 4

Summary of Observations for Primary Sample Objects

\begin{tabular}{|c|c|c|c|c|c|c|c|c|c|c|}
\hline No. & Name & Telescope & Mosaic & $\begin{array}{c}\text { Area } \\
\left(\mathrm{deg}^{2}\right)\end{array}$ & Astrometry & Photometry & $\langle X\rangle_{g}$ & $\langle X\rangle_{r}$ & $\begin{array}{l}T_{g} \\
(\mathrm{~s})\end{array}$ & $\begin{array}{l}T_{r} \\
(\mathrm{~s})\end{array}$ \\
\hline 1 & Sculptor & Clay & $4 \times 3$ & 1.13 & GAIA-DR1 & secondary & 1.05 & 1.05 & $5 \times 90$ & $5 \times 180$ \\
\hline 2 & Whiting 1 & CFHT & $1 \times 1$ & 0.95 & GAIA-DR 1 & secondary & 1.31 & 1.20 & $6 \times 300$ & $6 \times 300$ \\
\hline 3 & Segue 2 & CFHT & $1 \times 1$ & 1.02 & SDSS-DR7 & SDSS & 1.19 & 1.35 & $6 \times 350$ & $6 \times 350$ \\
\hline 4 & Fornax & Clay & $2 \times 2$ & 0.62 & GAIA-DR1 & secondary & 1.01 & 1.01 & $5 \times 90$ & $5 \times 180$ \\
\hline 5 & AM 1 & Clay & $1 \times 1$ & 0.21 & GAIA-DR1 & SDSS & 1.07 & 1.07 & $5 \times 90$ & $5 \times 180$ \\
\hline 6 & Eridanus & CFHT & $1 \times 1$ & 0.94 & USNO-B1 & secondary & 1.33 & 1.33 & $6 \times 270$ & $6 \times 270$ \\
\hline 7 & Palomar 2 & CFHT & $1 \times 1$ & 0.95 & USNO-B1 & secondary & 1.14 & 1.31 & $6 \times 450$ & $6 \times 450$ \\
\hline 8 & Carina & Clay & $4 \times 4$ & 2.16 & GAIA-DR1 & secondary & 1.15 & 1.15 & $5 \times 90$ & $5 \times 180$ \\
\hline 9 & NGC 2419 & CFHT & $1 \times 1$ & 1.02 & SDSS-DR7 & SDSS & 1.40 & 1.17 & $6 \times 450$ & $6 \times 450$ \\
\hline 10 & Koposov 2 & CFHT & $1 \times 1$ & 0.94 & SDSS-DR7 & SDSS & 1.28 & 1.26 & $6 \times 500$ & $6 \times 500$ \\
\hline 11 & UMa II & CFHT & $2 \times 2$ & 2.77 & GAIA-DR1 & SDSS & 1.39 & 1.39 & $11 \times 270$ & $11 \times 468$ \\
\hline 12 & Pyxis & Clay & $1 \times 1$ & 0.20 & GAIA-DR1 & SDSS & 1.16 & 1.14 & $5 \times 180$ & $5 \times 180$ \\
\hline 13 & Leo $\mathrm{T}$ & Clay & $1 \times 1$ & 0.21 & GAIA-DR1 & SDSS & 1.45 & 1.47 & $5 \times 180$ & $5 \times 180$ \\
\hline \multirow[t]{2}{*}{14} & \multirow[t]{2}{*}{ Palomar 3} & CFHT & $1 \times 1$ & 0.98 & GAIA-DR1 & SDSS & 1.27 & 1.42 & $6 \times 270$ & $6 \times 270$ \\
\hline & & Clay & $1 \times 1$ & 0.21 & GAIA-DR1 & SDSS & 1.18 & 1.17 & $5 \times 90$ & $5 \times 180$ \\
\hline 15 & Segue 1 & CFHT & $1 \times 1$ & 0.95 & GAIA-DR1 & SDSS & 1.19 & 1.37 & $6 \times 60$ & $6 \times 60$ \\
\hline 16 & Leo I & CFHT & $1 \times 1$ & 1.04 & GAIA-DR1 & SDSS & 1.25 & 1.10 & $11 \times 300$ & $11 \times 360$ \\
\hline 17 & Sextans & CFHT & $2 \times 2$ & 3.30 & GAIA-DR1 & SDSS & 1.10 & 1.16 & $6 \times 310$ & $6 \times 310$ \\
\hline 18 & UMa I & CFHT & $2 \times 1$ & 0.98 & GAIA-DR1 & SDSS & 1.24 & 1.21 & $6 \times 600$ & $6 \times 600$ \\
\hline 19 & Willman 1 & CFHT & $1 \times 1$ & 0.94 & GAIA-DR1 & SDSS & 1.40 & 1.37 & $6 \times 180$ & $6 \times 180$ \\
\hline 20 & Leo II & Clay & $2 \times 2$ & 0.15 & GAIA-DR1 & SDSS & 1.65 & 1.65 & $5 \times 90$ & $5 \times 180$ \\
\hline 21 & Palomar 4 & CFHT & $1 \times 1$ & 0.96 & GAIA-DR1 & SDSS & 1.24 & 1.31 & $6 \times 440$ & $6 \times 440$ \\
\hline 22 & Leo V & Clay & $1 \times 1$ & 0.21 & GAIA-DR1 & SDSS & 1.20 & 1.18 & $5 \times 90$ & $5 \times 180$ \\
\hline 23 & Leo IV & Clay & $1 \times 1$ & 0.21 & GAIA-DR1 & SDSS & 1.15 & 1.14 & $5 \times 90$ & $5 \times 180$ \\
\hline 24 & Koposov 1 & Clay & $1 \times 1$ & 0.21 & GAIA-DR1 & SDSS & 1.33 & 1.33 & $5 \times 90$ & $5 \times 180$ \\
\hline 25 & ComBer & CFHT & $2 \times 2$ & 3.51 & GAIA-DR1 & SDSS & 1.10 & 1.10 & $11 \times 270$ & $11 \times 468$ \\
\hline 26 & CVn II & CFHT & $1 \times 1$ & 1.01 & GAIA-DR1 & SDSS & 1.44 & 1.38 & $6 \times 380$ & $6 \times 380$ \\
\hline 27 & CVn I & CFHT & $1 \times 1$ & 0.96 & GAIA-DR1 & SDSS & 1.65 & 1.56 & $9 \times 300$ & $9 \times 450$ \\
\hline 28 & $\mathrm{AM} 4$ & Clay & $1 \times 1$ & 0.21 & GAIA-DR1 & SDSS & 1.04 & 1.03 & $5 \times 90$ & $5 \times 180$ \\
\hline 29 & Bootes II & CFHT & $1 \times 1$ & 0.97 & GAIA-DR1 & SDSS & 1.24 & 1.23 & $6 \times 400$ & $6 \times 400$ \\
\hline 30 & Bootes I & CFHT & $1 \times 2$ & 1.59 & GAIA-DR1 & SDSS & 1.30 & 1.30 & $6 \times 140$ & $6 \times 140$ \\
\hline 31 & NGC 5694 & CFHT & $1 \times 1$ & 0.95 & GAIA-DR1 & secondary & 1.45 & 1.45 & $6 \times 60$ & $6 \times 60$ \\
\hline 32 & Muñoz 1 & CFHT & $1 \times 1$ & $\ldots$ & GAIA-DR1 & SDSS & 1.54 & 1.50 & $6 \times 200$ & $6 \times 200$ \\
\hline 33 & NGC 5824 & Clay & $1 \times 1$ & 0.21 & GAIA-DR1 & SDSS & 1.08 & 1.06 & $5 \times 90$ & $5 \times 180$ \\
\hline 34 & Ursa Minor & CFHT & $2 \times 2$ & 3.21 & GAIA-DR1 & SDSS & 1.54 & 1.50 & $6 \times 200$ & $6 \times 200$ \\
\hline 35 & Palomar 14 & CFHT & $1 \times 1$ & 0.95 & GAIA-DR1 & SDSS & 1.33 & 1.25 & $6 \times 120$ & $6 \times 120$ \\
\hline 36 & Hercules & CFHT & $2 \times 1$ & 2.01 & GAIA-DR1 & SDSS & 1.11 & 1.14 & $6 \times 400$ & $6 \times 400$ \\
\hline 37 & NGC 6229 & CFHT & $1 \times 1$ & 0.91 & GAIA-DR1 & SDSS & 1.34 & 1.39 & $6 \times 90$ & $6 \times 90$ \\
\hline 38 & Palomar 15 & CFHT & $1 \times 1$ & 1.03 & GAIA-DR1 & SDSS & 1.09 & 1.13 & $6 \times 2250$ & $6 \times 225$ \\
\hline 39 & Draco & CFHT & $2 \times 2$ & 3.34 & GAIA-DR1 & SDSS & 1.48 & 1.37 & $6 \times 150$ & $6 \times 125$ \\
\hline 40 & NGC 7006 & CFHT & $1 \times 1$ & 0.95 & GAIA-DR1 & SDSS & 1.01 & 1.01 & $6 \times 240$ & $6 \times 240$ \\
\hline 41 & Segue 3 & Clay & $1 \times 1$ & 0.21 & GAIA-DR1 & SDSS & 1.72 & 1.65 & $5 \times 90$ & $5 \times 180$ \\
\hline 42 & Pisces II & Clay & $1 \times 1$ & 0.21 & GAIA-DR1 & SDSS & 1.23 & 1.26 & $5 \times 225$ & $5 \times 450$ \\
\hline 43 & Palomar 13 & CFHT & $1 \times 1$ & 0.98 & GAIA-DR1 & SDSS & 1.05 & 1.01 & $6 \times 360$ & $6 \times 360$ \\
\hline \multirow[t]{2}{*}{44} & NGC 7492 & CFHT & $1 \times 1$ & 0.94 & GAIA-DR1 & secondary & 1.23 & 1.23 & $6 \times 120$ & $6 \times 120$ \\
\hline & & Clay & $1 \times 1$ & 0.21 & GAIA-DR1 & secondary & 1.07 & 1.09 & $5 \times 90$ & $5 \times 180$ \\
\hline
\end{tabular}



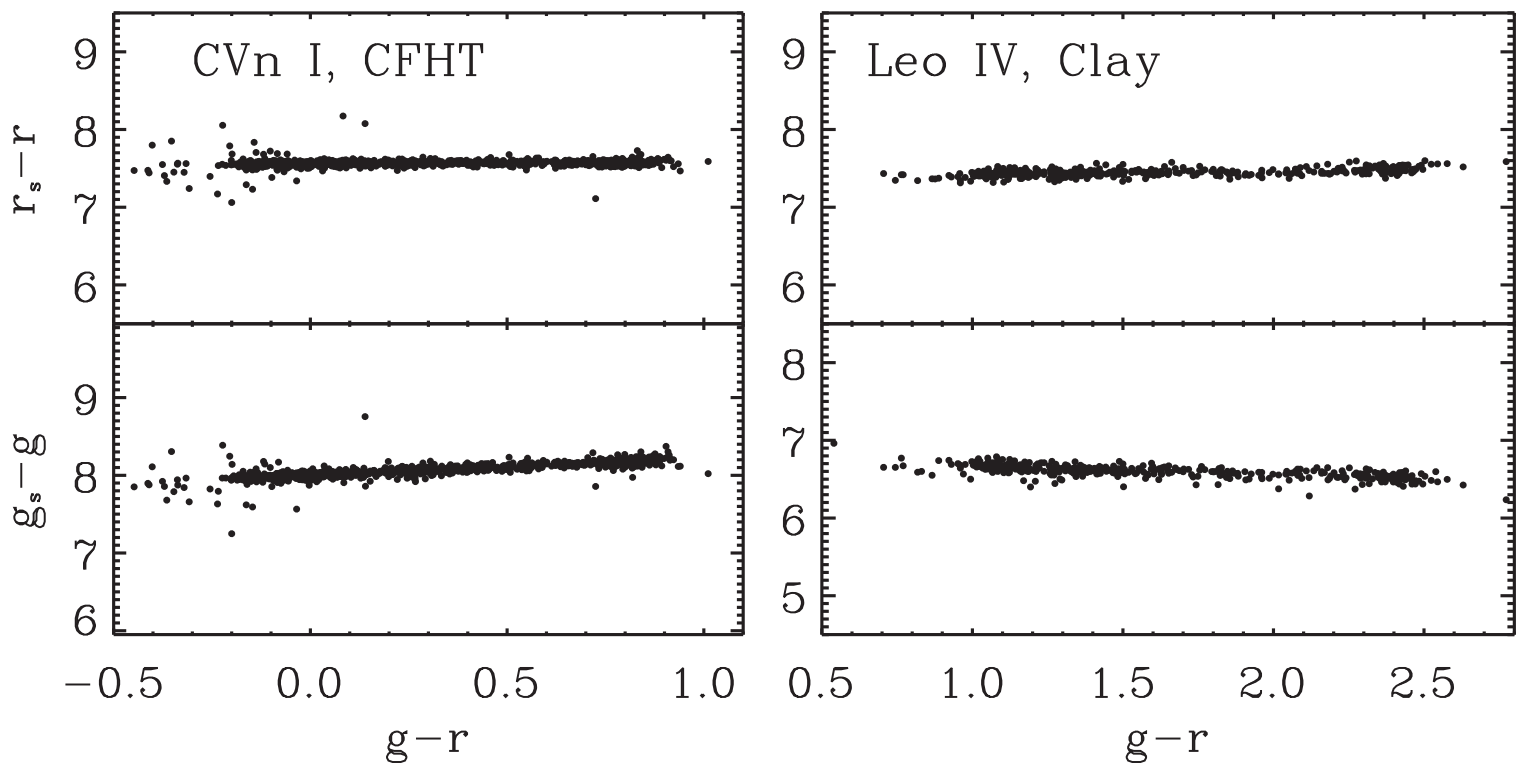

Figure 3. Left panels: difference between the CFHT instrumental and SDSS calibrated magnitudes as a function of $(g-r)$ color for stellar sources in CVn I. This comparison includes data from all 36 chips, for objects having $g$ - and $r$-band SDSS magnitudes in the range 18-21.5. Right panels: similar to the previous panel, except for stars in Leo IV as observed by the Clay telescope.

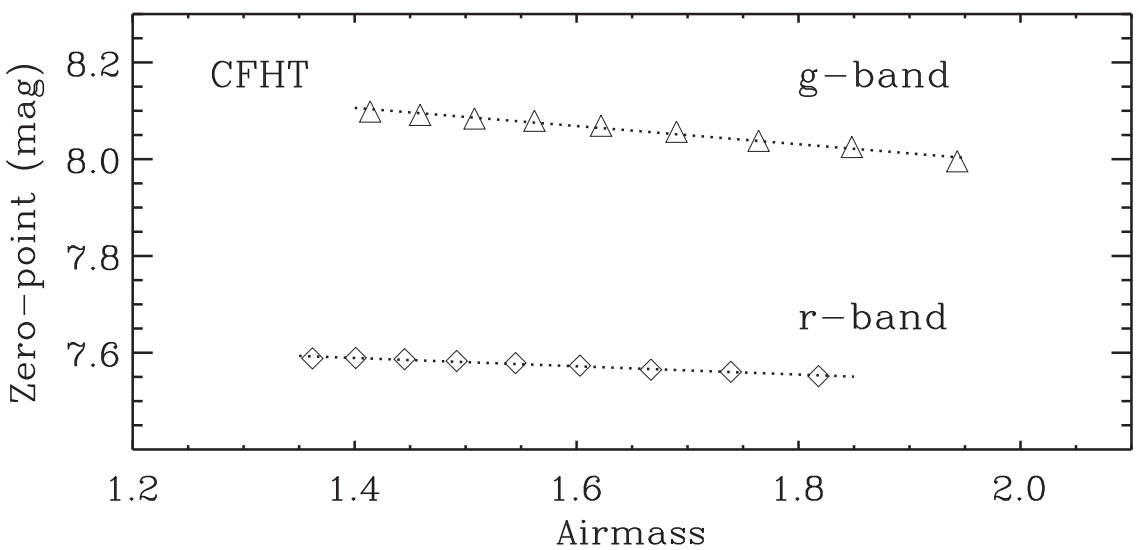

Figure 4. Photometric zero-point plotted as a function of airmass for CFHT observations of CVn I. The upper and lower symbols show the trends observed in the $g$ and $r$-bands, respectively.

\subsection{Clay-Megacam Imaging}

For 16 additional targets, including Pal 3 and NGC 7492, in the southern hemisphere, Clay-Megacam imaging was acquired during eight nights on the $6.5 \mathrm{~m}$ Clay telescope in 2010 November and 2011 April. Clay-Megacam is a large mosaic CCD camera that also consists of 36 CCDs $(2048 \times 4608$ pixels) but at a scale of 0 "! 08 pixel $^{-1}$ (McLeod et al. 2015). This array provides instantaneous coverage of a $0.4 \times 0.4$ field.

Because this field of view is about five times smaller than that of its northern counterpart, we used multiple pointings for the most extended objects; in the case of Carina, we cover an area of $2.6 \mathrm{deg}^{2}$ in 16 different fields. For the more compact objects - usually globular clusters - only a single pointing was needed, with the target positioned at the center of the mosaic. To maintain survey homogeneity, Clay-Megacam images were also taken in the Sloan $g$ and $r$ filters. In all cases, we collected five dithered exposures per pointing in each filter to cover chip gaps. Images were usually acquired in dark time and during seeing conditions comparable to those at CFHT $(0$ ". $7-1$ !" 1$)$. Excluding the two targets that appear in both our CFHT and Clay programs (see below), the Clay imaging covers a total area of $8.75 \mathrm{deg}^{2}$. In all, our MegaCam imaging covers a combined area of $52 \mathrm{deg}^{2}$.

\subsection{Image Processing and Astrometric Calibration}

Data from both instruments used in our primary survey were pre-processed prior to delivery. For CFHT-MegaCam, preprocessing was done by the CFHT staff using the standard Elixir package, while the Clay-Megacam data were pre-reduced at the HarvardSmithsonian Center for Astrophysics (CfA). In both cases, the goal of preprocessing is to provide the user with frames that are corrected for the instrumental signature across the mosaic. This involves bad pixel correction, bias subtraction, flat-fielding, and the calculation of preliminary astrometric and photometric solutions that are included in the headers of the pre-processed images.

However, the World Coordinate System (WCS) information provided with the processed data is only approximate. For both the Clay and CFHT data, we therefore refine the astrometric solution using the latest freely available SCAMP $^{7}$ package

7 http://astromatic.net/software/scamp/ 
Table 5

Photrometry of Individual Objects

\begin{tabular}{|c|c|c|c|c|c|c|c|c|}
\hline Star ID & R.A.(deg, 2000.0) & Decl.(deg, 2000.0) & $g$ & $\sigma_{g}$ & $r$ & $\sigma_{r}$ & chi & Sharp \\
\hline $\mathrm{scl}-1$ & 15.067787 & -33.383965 & 20.9255 & 0.0023 & 20.4028 & 0.0018 & 0.5097 & 0.0035 \\
\hline $\mathrm{scl}-2$ & 15.102280 & -33.383710 & 23.2864 & 0.0119 & 23.0176 & 0.0094 & 0.4387 & -0.0646 \\
\hline $\mathrm{scl}-3$ & 15.101350 & -33.382940 & 23.3046 & 0.0121 & 22.9910 & 0.0092 & 0.4393 & 0.0703 \\
\hline $\mathrm{scl}-4$ & 15.098076 & -33.382812 & 22.9103 & 0.0094 & 22.4541 & 0.0068 & 0.4669 & 0.6238 \\
\hline
\end{tabular}

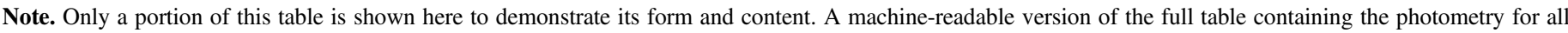
primary objects is available. Objects from different satellites can be separated by their ID name.

(This table is available in its entirety in machine-readable form.)

Table 6

Summary of Observations for Secondary Sample Objects

\begin{tabular}{|c|c|c|c|c|c|c|c|c|c|c|}
\hline No. & Name & Telescope & Mosaic & $\begin{array}{c}\text { Area } \\
\left(\mathrm{deg}^{2}\right)\end{array}$ & Astrometry & Photometry & $\overline{\langle X\rangle_{g}}$ & $\langle X\rangle_{r}$ & $\begin{array}{l}T_{g} \\
\text { (s) }\end{array}$ & $\begin{array}{c}T_{r} \\
\text { (s) }\end{array}$ \\
\hline 1 & Triangulum II & LBT & $1 \times 1$ & $23^{\prime} \times 25^{\prime}$ & PS1 & PS1 & $\ldots$ & $\ldots$ & $6 \times 200$ & $6 \times 200$ \\
\hline 2 & Eridanus 3 & Blanco & $1 \times 1$ & $\sim 3$ & DESDM & secondary & 1.09 & 1.10 & $1 \times 90$ & $1 \times 90$ \\
\hline 3 & Horologium I & Blanco & $1 \times 1$ & $\sim 3$ & DESDM & secondary & 1.19 & 1.40 & $1 \times 90$ & $1 \times 90$ \\
\hline 4 & Horologium II & Blanco & $1 \times 1$ & $\sim 3$ & DESDM & APASS DR8 & $\ldots$ & $\cdots$ & $1 \times 90$ & $1 \times 90$ \\
\hline 5 & Reticulum II & Blanco & $1 \times 1$ & $\sim 3$ & DESDM & secondary & 1.38 & 1.39 & $1 \times 90$ & $1 \times 90$ \\
\hline 6 & Eridanus II & Blanco & $1 \times 1$ & $\sim 3$ & DESDM & secondary & 1.31 & 1.32 & $1 \times 90$ & $1 \times 90$ \\
\hline 7 & Pictoris I & Blanco & $1 \times 1$ & $\sim 3$ & DESDM & secondary & 1.09 & 1.10 & $1 \times 90$ & $1 \times 90$ \\
\hline 8 & Crater & PS1 & $\cdots$ & $\cdots$ & PS1 & PS1 & $\cdots$ & $\cdots$ & $5 \times 90$ & $5 \times 180$ \\
\hline 9 & Hydra II & Blanco & $1 \times 1$ & $\sim 3$ & DESDM & $\cdots$ & $\cdots$ & $\cdots$ & $3 \times 267$ & $3 \times 267$ \\
\hline 10 & Indus 1 & Blanco & $1 \times 1$ & $\sim 3$ & DESDM & secondary & 1.20 & 1.07 & $1 \times 90$ & $1 \times 90$ \\
\hline 11 & Balbinot 1 & CFHT & $1 \times 1$ & $\sim 1$ & $\ldots$ & SDSS & $\ldots$ & $\ldots$ & $6 \times 467$ & $6 \times 633$ \\
\hline 12 & Kim 1 & Blanco & $1 \times 1$ & $\sim 3$ & DESDM & SDSS & $\ldots$ & $\cdots$ & $8 \times 210$ & $5 \times 210$ \\
\hline 13 & Grus I & Blanco & $1 \times 1$ & $\sim 3$ & DESDM & secondary & 1.10 & 1.12 & $1 \times 90$ & $1 \times 90$ \\
\hline 14 & Phoenix 2 & Blanco & $1 \times 1$ & $\sim 3$ & DESDM & secondary & 1.17 & 1.38 & $1 \times 90$ & $1 \times 90$ \\
\hline
\end{tabular}

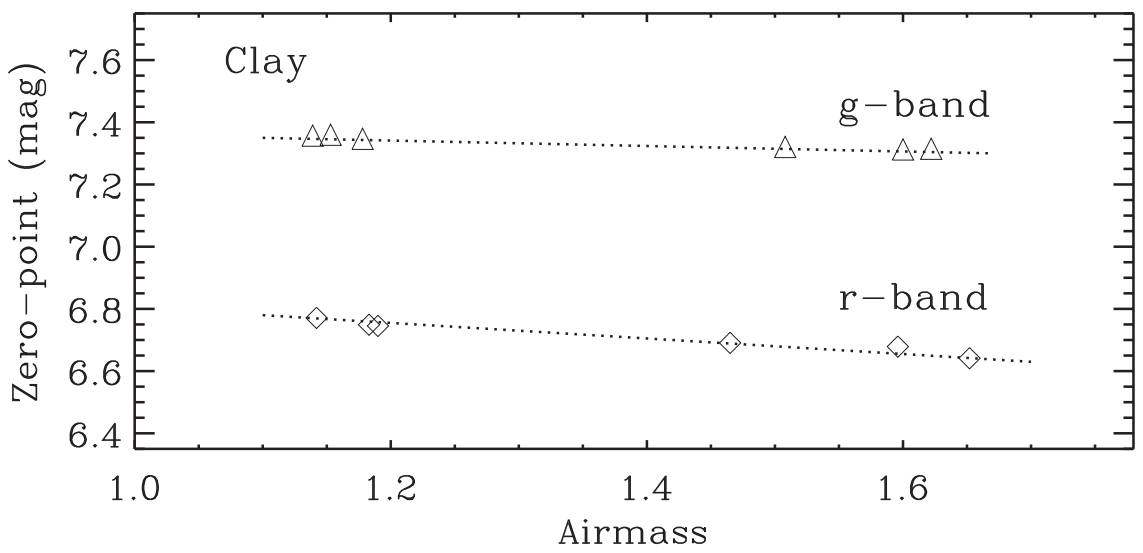

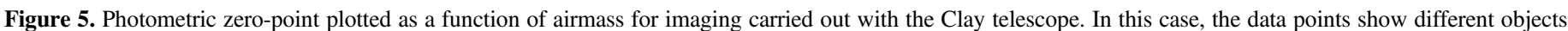
observed using the same exposure time. As in the previous figure, the upper and lower symbols show the trends in $g$ - and $r$-bands, respectively.

(Bertin 2006). First, Terapix SExtractor ${ }^{8}$ (Bertin \& Arnouts 1996) was run on all chips (SCAMP reads in the output files generated by SExtractor) and output files were written in the FITS-LDAC format (where LDAC $=$ Leiden Data Analysis Center). SCAMP was then run on all chips separately. SCAMP uses the approximate WCS information in the frames' headers as a starting point, and then computes astrometric solutions using external reference catalogs. In our case, we used the GAIA (DR1, Gaia collaboration et al. 2016) catalog for 39

\footnotetext{
$\overline{8 \mathrm{http}: / / \text { astromatic.net/software/sextractor/ }}$
}

objects for which the combination of spatial density and magnitude overlap yielded enough stars in common to determine a reliable solution. For the other five objects, the solutions based on GAIA were not precise enough due to the low number of stars per chip in common, thus we used the SDSS-Data Release 7 (DR7, Abazajian et al. 2009) for three of them present in SDSS, and used the USNO-B1 catalog for the remaining two targets that fall outside the SDSS footprint. CFHT-MegaCam chips are five times larger in terms of sky coverage than those of Clay-Megacam, so we found systematically more stars in common between each of our chips and 


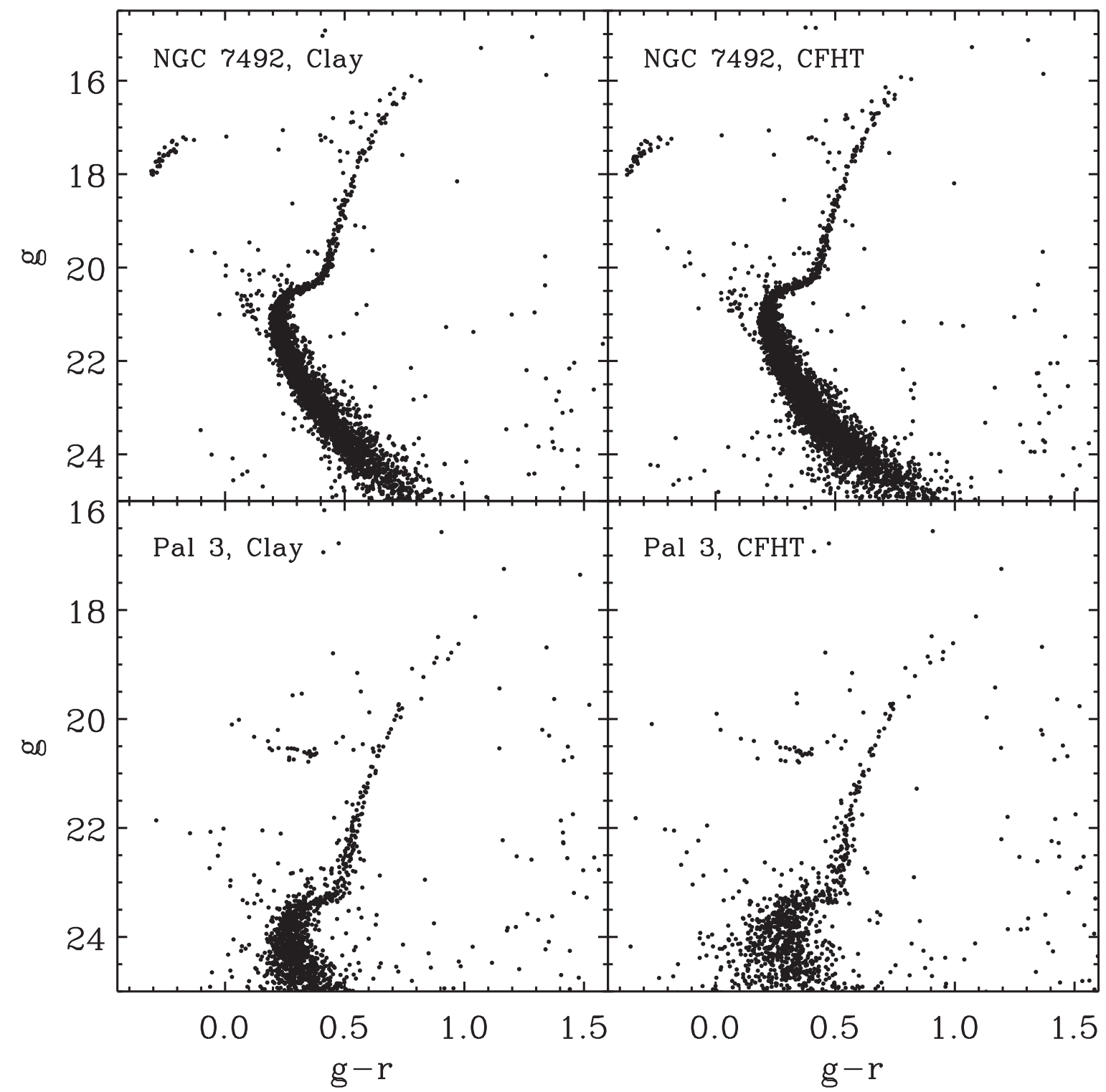

Figure 6. Comparison of the color-magnitude diagrams for two of our program objects, NGC 7492 and Pal 3, observed from both hemispheres. The left and right panels show data within the half-light radii obtained with Clay and CFHT, respectively. The Clay-Megacam data appear deeper, showing narrower sequences at fainter magnitudes.

the reference catalog for the CFHT data. Generally speaking, a couple hundred stars were used to compute the astrometric solution for CFHT chips, while several tens of stars were typically used for the case of Clay data. Despite the difference in sample size, these are sufficient in both cases to avoid significant shot-noise, thus our astrometric uncertainties do not depend on the instrument but on the reference catalog used for each object. For objects where GAIA was used, we typically obtained global astrometric uncertainties of rms $\sim 0$ "'04-0." 06 , with internal accuracy typically better than $\sim 0{ }^{\prime \prime} 02$. For those in SDSS, we obtained rms $\sim 0$ ". $10-0$ ". 20 and for those objects in which we used USNO-B1 as the reference catalog, typical rms uncertainties in the astrometry were $\sim 0$ !" 3 .

The output from SCAMP is a single FITS header file per processed frame. For the CFHT-MegaCam images, this SCAMP output was used to update the WCS information for each chip. Point-source photometry was then performed on the images with the updated headers (see Section 3.4). The final photometry was then used to translate $x$ and $y$ stellar positions into equatorial coordinates using the astrometric solution coefficients in the image headers.

In the case of the Clay-Megacam images, the celestial projection used by the CfA team to determine the preliminary astrometric solution is zenithal polynomial. Unfortunately, this projection is incompatible with the current version of SCAMP, so the images were reprojected using REMAP into a tangential projection (which is SCAMP compatible). There is, however, an uncertainty involved in translating a given pixel value from one projection to another-a process that introduces small but noticeable differences in the magnitudes obtained in the subsequent photometry. Therefore, we carried out the photometry in the images with the original zenithal polynomial projection. The $x$ and $y$ positions of stars in the catalogs were then translated into $x^{\prime}$ and $y^{\prime}$ positions corresponding to the same star in the image reprojected into a tangential projection. Finally, these tangential $x^{\prime}$ and $y^{\prime}$ positions were transformed 


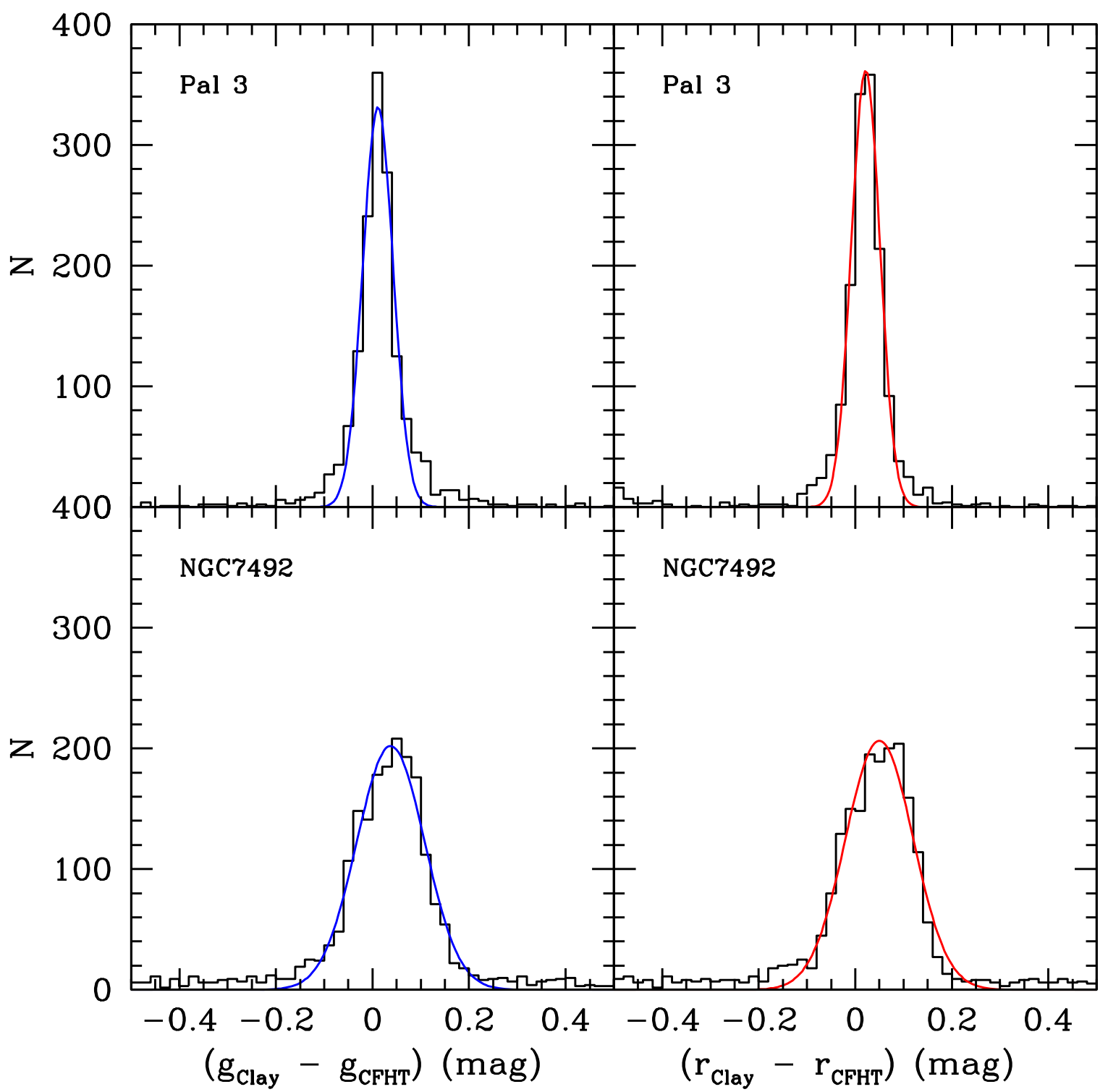

Figure 7. Histograms showing the difference in $g$ - and $r$-band magnitude for stellar sources in Pal 3 (upper panels) and NGC 7492 (lower panels), both of which were observed from Clay and CFHT The smooth curve in each panel shows the best-fit Gaussian distribution.

into equatorial coordinates using the WCS information obtained using SCAMP in the tangential reprojected image.

\subsection{Point-spread Function (PSF) Photometry}

The photometric processing was similar for images from both telescopes. Prior to carrying out point-source photometry on our data, we split each mosaic frame into its 36 individual chips. We then performed PSF photometry by first running DAOPHOT/ALLSTAR on the individual (non-coadded) frames and then running ALLFRAME on the resulting files, as detailed in Stetson (1994).

ALLFRAME performs photometry simultaneously on all $g$ and $r$ frames for a given field. DAOPHOT/ALLSTAR must be run prior to ALLFRAME in order to determine PSF solutions for each chip, and to generate starlists for individual frames. The optimum starlists that are needed as inputs to ALLFRAME were generated by cross-matching the DAOPHOT/ALLSTAR results for the individual frames using the DAOMATCH and
DAOMASTER packages (Stetson 1993). These packages also provide reasonably good estimates of the spatial offsets between dithered individual exposures necessary to run ALLFRAME. Final output files from ALLFRAME were then combined into a single master catalog for each program object.

\subsection{Photometric Calibration: Objects in Common with SDSS}

For objects that fall inside the SDSS footprint, our instrumental magnitudes have been calibrated through a direct comparison to the SDSS-DR7. First, we matched our photometric catalog for each object with the SDSS stellar catalog, typically finding several hundred stars per chip in common with the SDSS. To determine zero-points and color terms, we used only SDSS stars with $18<r_{\text {SDSS }}<21.5$ and $18<g_{\text {SDSS }}<22$. The faint limit was chosen to eliminate stars from SDSS with large photometric uncertainties and the bright limit was chosen to avoid saturated stars in our MegaCam data. 


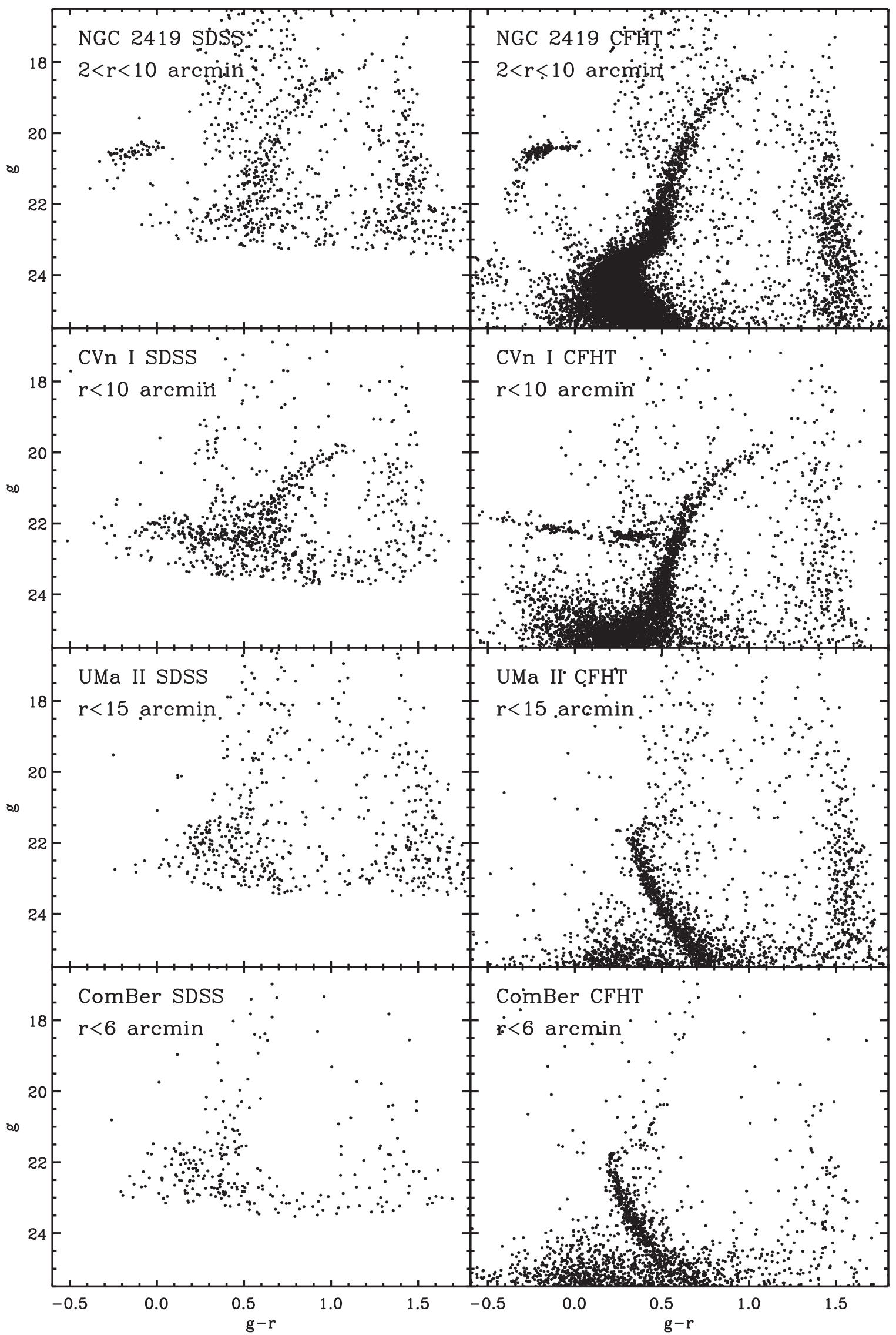

Figure 8. Color-magnitude diagrams for four of our program objects: NGC 2419, CVn I, UMa II, and ComBer. The panels on the left show CMDs based on data from SDSS (DR12), while those on the right show our new CFHT and Clay photometry. 

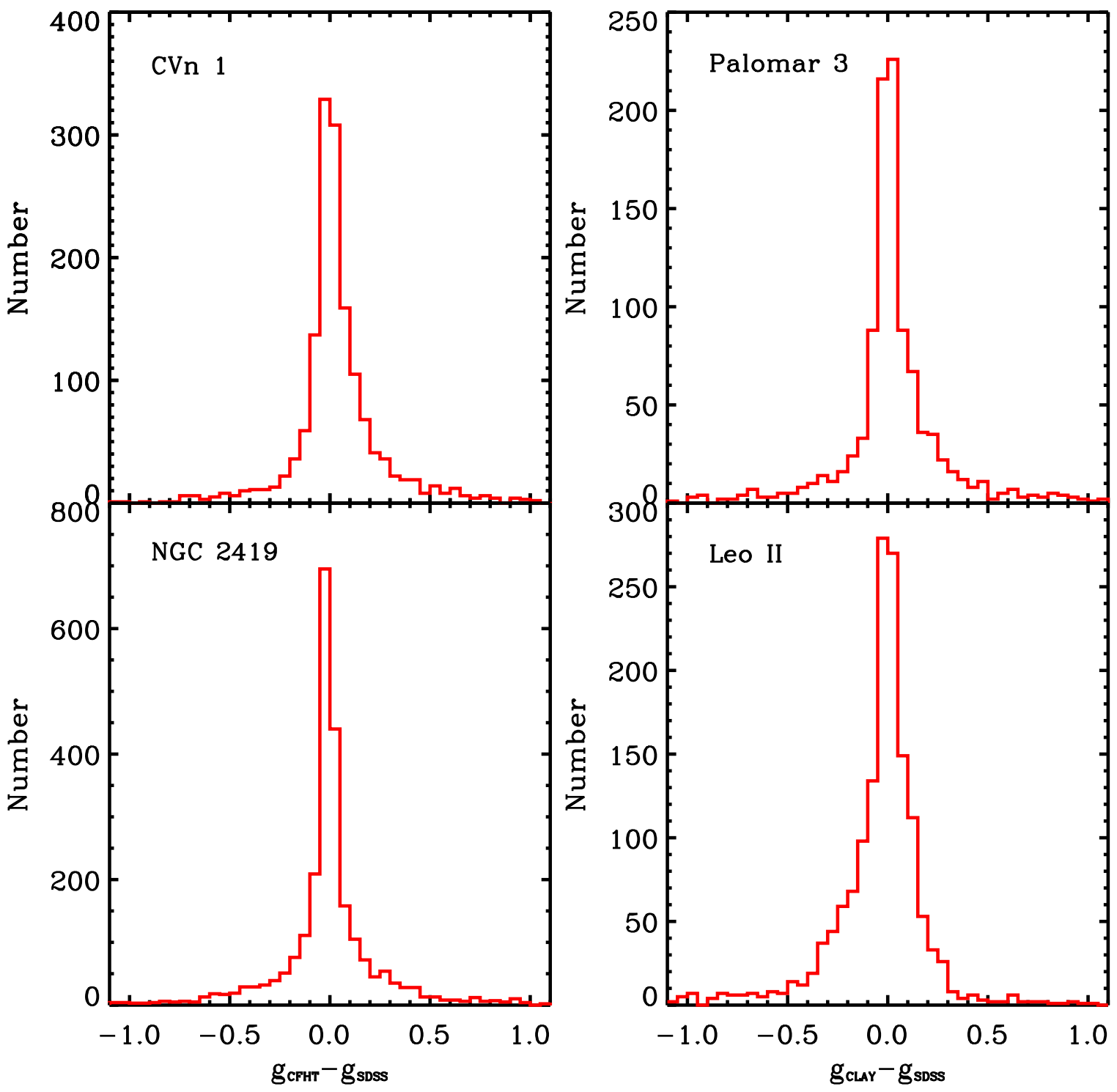

Figure 9. (Upper left panel) Histogram of the difference between the $g$-band magnitude from the CFHT and the SDSS catalogs for CVn 1. (Lower left panel) Same as above but for NGC 2419. (Upper right panel) Histogram of the difference between the $g$-band magnitude from the Clay and the SDSS catalogs for Palomar 3. (Lower right panel) Same as above but for Leo II.

We then used the matched catalog to fit equations of the form:

$$
\begin{aligned}
& g_{\mathrm{SDSS}}=g+g_{0}+g_{1}(g-r) \\
& r_{\mathrm{SDSS}}=r+r_{0}+r_{1}(g-r) .
\end{aligned}
$$

Here, $g$ and $r$ are our instrumental magnitudes, $g_{0}$ and $r_{0}$ are the zero-points and $g_{1}$ and $r_{1}$ are the color terms. Because we are calibrating directly to SDSS photometry, we do not need to determine the airmass terms.

In their CFHT-MegaCam study of Coma Berenices and Ursa Major II, Muñoz et al. (2010) derived zero-points and color terms for each chip individually, in order to examine possible chip-to-chip variations for this instrument. In both cases, they found that the chip-to-chip differences, for both the zero-points and color terms, were smaller than the uncertainties in the derived parameters. For this study, we repeated this test using CVn I and Segue 1 and found similar results. Unfortunately, for the Clay-Megacam data, we could not carry out the same test given the low number of stars per chip in common with SDSS (i.e., all the Clay targets that fall within the SDSS footprint are ultra-faint dwarf galaxies with low stellar counts). As an alternative, we used stars in the overlapping regions between chips to assess whether there were systematic chip-to-chip variations. For these stars, we found that the average magnitude difference between the chips was always smaller than the magnitude uncertainties of our stars. For each satellite, we therefore combined stars from all 36 chips to derive global zero-point and color-term values via a linear least-squares fit (weighting by the respective uncertainties in the ALLFRAME magnitudes and rejecting $3 \sigma$ outliers).

We calculated zero-points and color terms for each mosaic field independently. For the CFHT-MegaCam calibration, uncertainties in the zero-points were typically $0.003-0.004 \mathrm{mag}$. While the $g_{0}^{\prime}$ and $r_{0}^{\prime}$ terms are a function of exposure time and airmass, the color terms remain fairly constant for all objects, with variations of less than $2 \%$. For the Clay-Megacam calibration, typical uncertainties in the zero-points are 0.002-0.009 mag. The color terms showed object-to-object variations of less than 5\%. A comparison between 

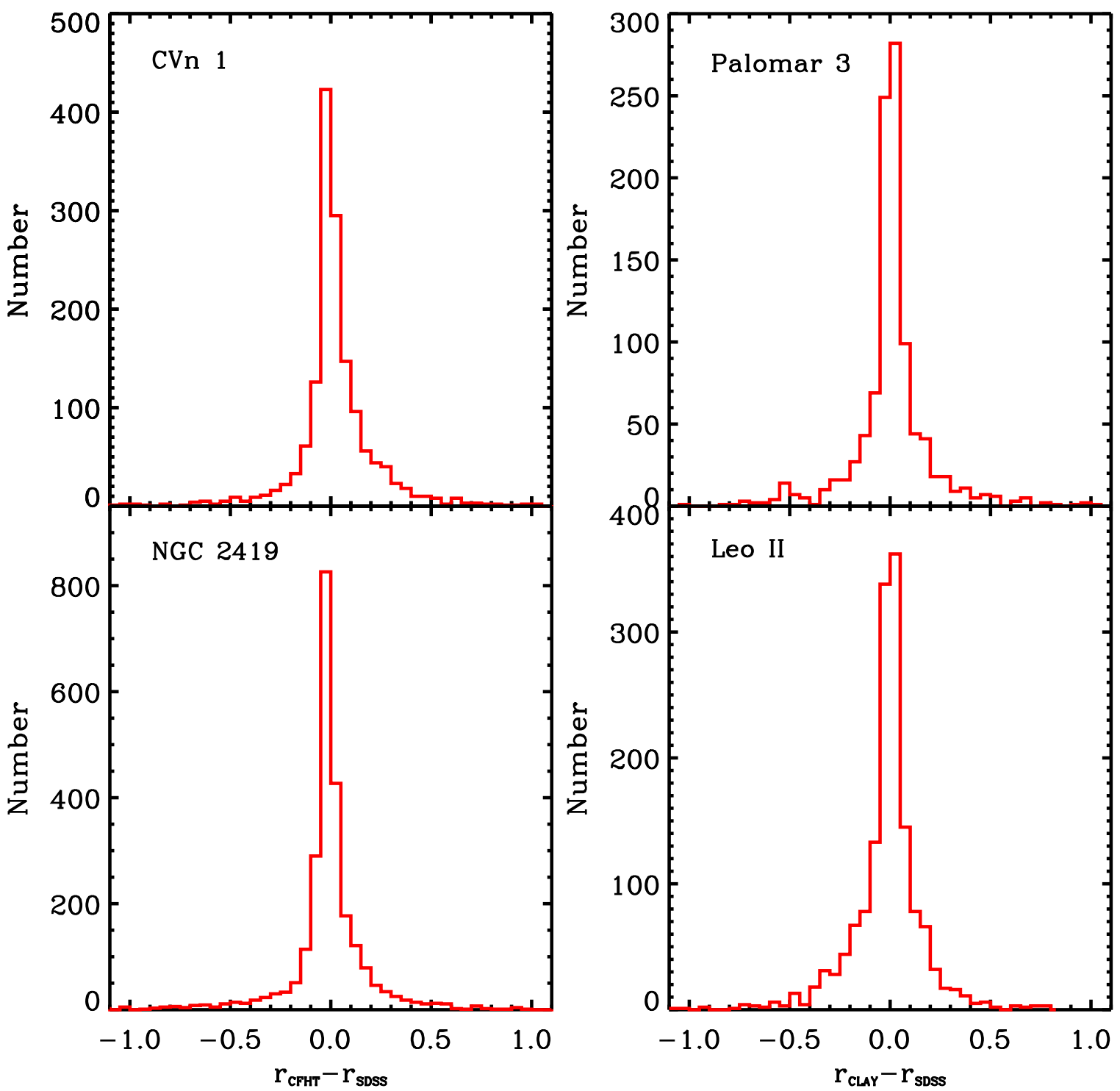

Figure 10. Same as Figure 9 but for $r$-band magnitudes.

the CFHT and SDSS magnitudes used to calibrate our photometry is shown in Figure 3 for two representative stellar systems: CVn I (CFHT) and Leo IV (Clay).

\subsection{Photometric Calibration: Objects Not in Common with SDSS}

Some objects in our primary sample fall outside the SDSS footprint and therefore require a different method of calibration. In such cases, the instrumental magnitudes were calibrated by applying the following equation:

$$
\begin{aligned}
& g_{\mathrm{SDSS}}=g+g_{0}+g_{1}(g-r)+g_{2} X \\
& r_{\mathrm{SDSS}}=r+r_{0}+r_{1}(g-r)+r_{2} X .
\end{aligned}
$$

where $g_{2}$ and $r_{2}$ are the airmass terms, and $X$ is the airmass.

The full set of coefficients $g_{0}, g_{1}, g_{2}, r_{0}, r_{1}$, and $r_{2}$ were determined using the photometry of objects in SDSS as secondary standards. The color terms derived for CFHT were $\left\langle g_{1, \mathrm{CFHT}}\right\rangle=0.203 \pm 0.003$ and $\left\langle r_{1, \mathrm{CFHT}}\right\rangle=0.021 \pm 0.002$, while for Clay, we obtained $\left\langle g_{1, \text { Clay }}\right\rangle=-0.098 \pm 0.010$ and $\left\langle r_{1, \text { Clay }}\right\rangle=0.052 \pm 0.005$.
To determine the airmass terms, we calculated the variation of the zero-points as a function of airmass for objects in the SDSS. Figure 4 shows the $g$ - and $r$-band zero-points obtained for a variety of airmasses and the linear trends fitted to them:

$$
\begin{aligned}
& g_{2, \text { CFHT }}=-0.176 \pm 0.015 \\
& r_{2, \text { CFHT }}=-0.080 \pm 0.007 .
\end{aligned}
$$

To derive the Clay airmass terms, we used different objects at different airmasses, taking advantage of the fact that most of the objects observed at Clay had the same exposure time. In particular, we used those objects having the lowest photometric errors in the Clay sample: Leo IV, Leo V, Palomar 3, Segue 1, and two different fields for Leo II. Figure 5 shows zero-points obtained for these systems at different airmasses. The best-fit linear trends yield airmass terms of

$$
\begin{aligned}
& g_{2, \text { Clay }}=-0.222 \pm 0.011 \\
& r_{2, \text { Clay }}=-0.094 \pm 0.009 .
\end{aligned}
$$

Lastly, we determined zero-points $g_{0}$ and $r_{0}$. For our Clay targets, this was carried out simultaneously with the 

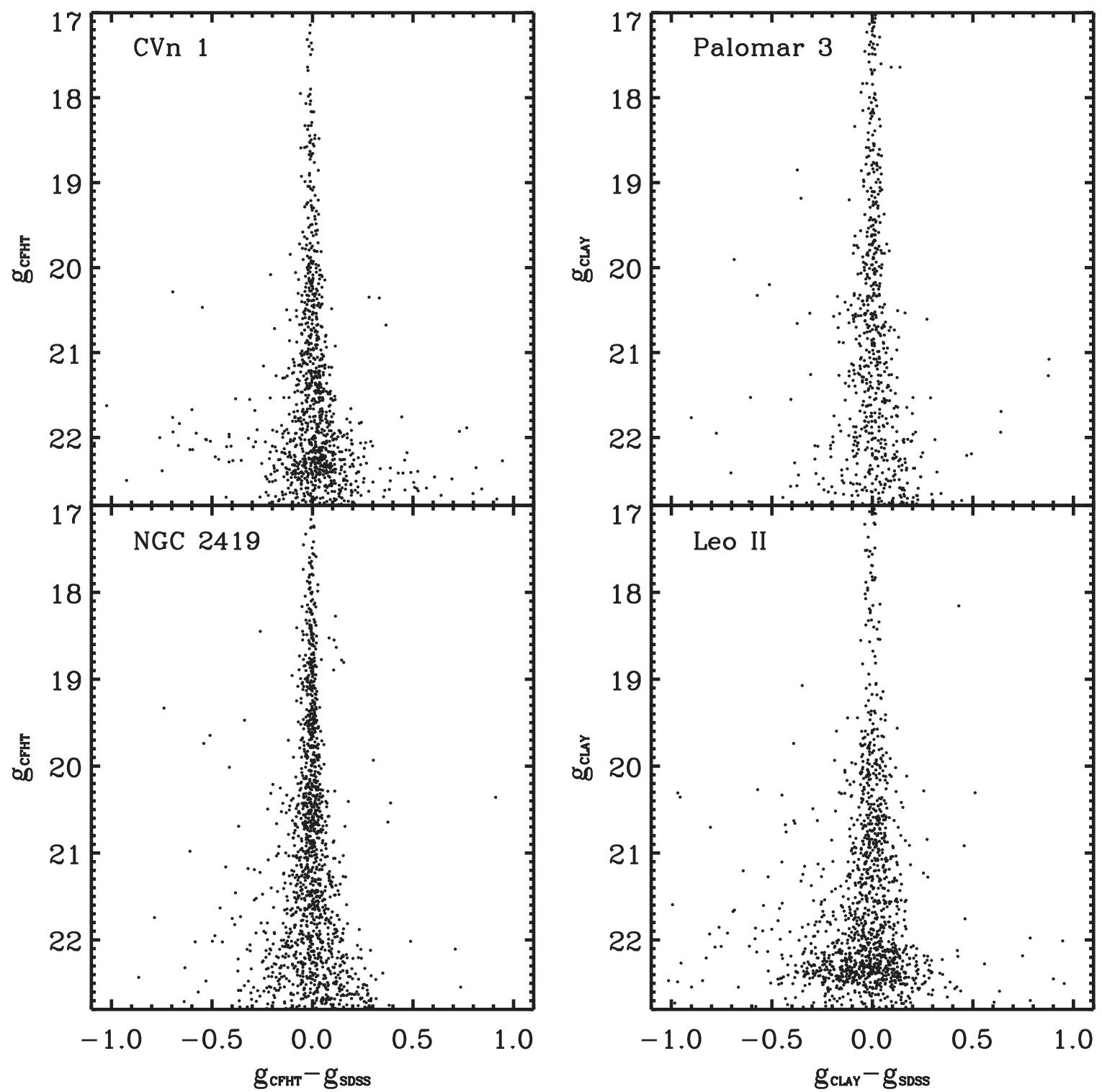

Figure 11. (Upper left panel) Difference in $g$-band magnitudes between CFHT and SDSS data vs. CFHT $g$-band magnitude for CVn 1. (Lower left panel) Same as above but for NGC 2419. (Upper right panel) Difference in $g$-band magnitudes between CFHT and SDSS data vs. CFHT g-band magnitude for Palomar 3. (Lower right panel) Same as above but for Leo II.

measurement of the extinction terms since the exposure times were the same. The resulting zero-points were found to be:

$$
\begin{aligned}
& g_{0, \text { Clay }}=7.016 \pm 0.014 \\
& r_{0, \text { Clay }}=7.463 \pm 0.011 .
\end{aligned}
$$

Meanwhile, for the CFHT observations, the zero-point variations with exposure time (after correcting for airmass) had to be computed explicitly since, in this case, the images were taken over a fairly wide range in exposure time. For objects in common with the SDSS we found:

$$
\begin{aligned}
& g_{0, \text { CFHT }}=1.476+2.5 \log T_{\exp } \\
& r_{0, \text { CFHT }}=1.014+2.5 \log T_{\exp } .
\end{aligned}
$$

Using these relations, zero-points were then calculated for the remaining objects using their respective exposure times.
Table 5 presents the full catalogs for all 44 primary targets. The table includes the 2000.0 equatorial coordinates, and calibrated, unreddened $g$ and $r$ magnitudes, as well as their uncertainties. We also include the DAOPHOT chi and sharp parameters. We removed the majority of spurious and nonstellar detections by applying the following cut: $-0.5<$ sharp $<0.5$ and chi $<3$. Stars from different objects can be distinguished by their ID name.

\section{Imaging and Data Reductions for Secondary Targets}

As detailed in Section 2.2, in addition to the 44 objects observed with the Megacam imagers, we include 14 objects discovered after the completion of our observing campaign. For eight satellites discovered using DECam data from the DES survey (Koposov et al. 2015a; The DES Collaboration et al. 2015), Eridanus II and 3, Horologium I, Reticulum II, Pictoris I, Indus 1, Grus I, and Phoenix 2, 


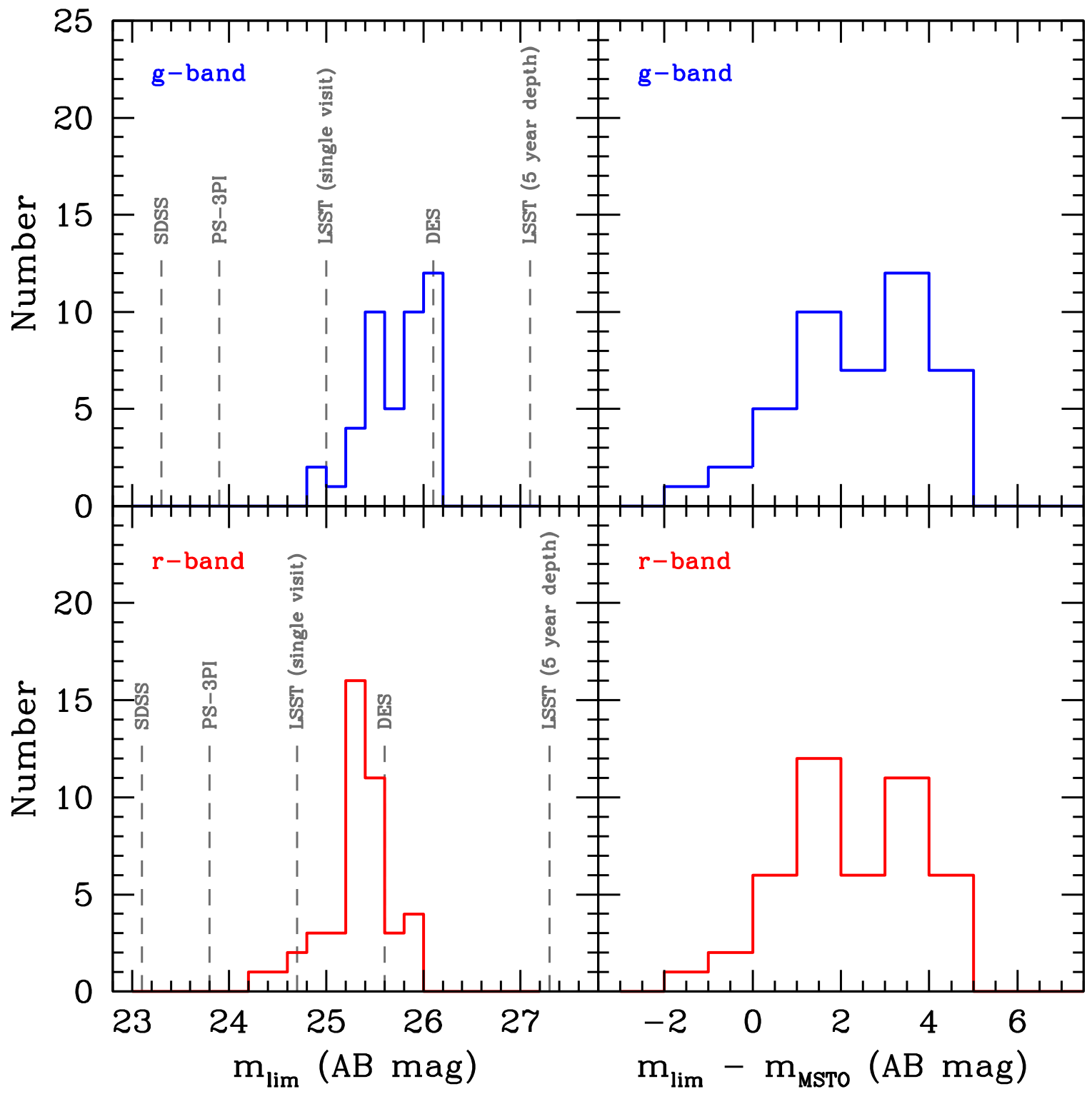

Figure 12. Upper left panel: distribution of limiting magnitude ( $5 \sigma$, point-source limits) for our 44 CFHT or Clay program objects. For comparison, the dashed vertical lines show the corresponding limits from a number of other notable surveys: i.e., Sloan Digital Sky Survey (SDSS), the Pan-STARRS 3PI survey, a single visit from LSST, the coadded Dark Energy Survey (DES), and the 5 year, coadded depth from LSST. Lower left panel: same as above, except for the $r$-band. Upper right panel: difference between our limiting magnitude and the main-sequence turnoff magnitude, $m_{\mathrm{MSTO}}$, for the same sample of 44 program objects. Our CFHT and Clay images reach a median depth $\simeq 2.2 \mathrm{mag}$ below the main-sequence turnoff in both bands. Lower right panel: same as above, except for the $r$-band.

we retrieved archival DECam data from the NOAO Science archive. ${ }^{9}$ In the case of Indus 1, discovered independently, we also obtained Kim's et al. photometry. We obtained the photometry for two other satellite candidates, Peg 3 (Kim et al. 2015a) and Tucana 2 (from the DES sample), but unfortunately our method for retrieving structural parameters was not able to converge due to the low number of stars in the files, therefore we do not include them in the final list. Table 6 presents a summary of observations for the secondary targets.

The archival data used in this catalog consisted, in most cases, of one DECam pointing observed in both the $g$ - and $r$-bands. The DECam imager consists of $622048 \times 4096$ pixel chips with a pixel scale of 0.2626 arcsec/pixel covering a total area of $3 \mathrm{deg}^{2}$. In some cases (Horo I, Ret II, Eri II), a

\footnotetext{
http://www.portal-nvo.noao.edu/
}

second DECam pointing overlapping the position of the satellite was present in the archives and thus both pointings were reduced together and combined to cover the chip gaps. In all cases, the exposure times were $90 \mathrm{~s}$. The subsequent photometry procedure was similar to that carried out for the Megacam imagers, i.e., DAOPHOT, ALLSTAR were performed in all the individual images and ALLFRAME was performed in the cases where more than one observation per field was used.

Equatorial coordinates for all objects detected by the DAOPHOT/ALLSTAR routines were obtained using the WCS information provided in the image headers. Comparison between stellar detections present in multiple observations of the same field showed that the internal precision was better than 0.1 arcsec.

To calibrate the instrumental photometry we used DECam data taken for a different program in the same bands. From our 

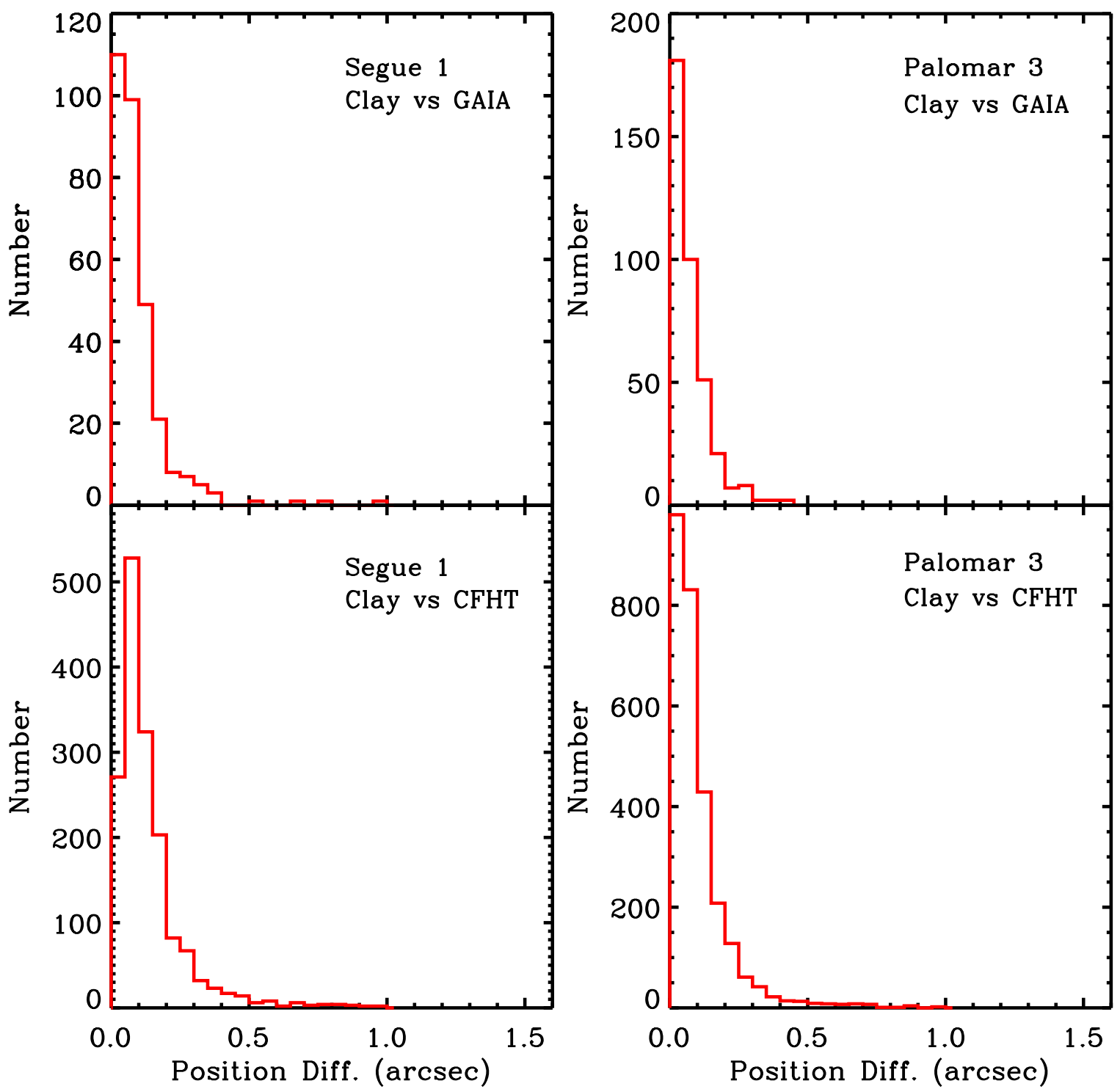

Figure 13. (Upper left panel) Histogram of the difference between the equatorial positions of the same objects in the Segue 1 field taken from the Clay and SDSS catalogs. (Lower left panel) Same as above but this time for objects from the Clay and CFHT data sets. (Upper right panel) Same as upper left panel but for Palomar 3. (Lower right panel) Same as above but for Palomar 3.

own DECam data we estimated the zero-points and color terms to be

$$
\begin{aligned}
& g_{0, \text { DECam }}=4.960 \pm 0.031 \\
& r_{0, \text { DECam }}=5.010 \pm 0.024
\end{aligned}
$$

and

$$
\begin{aligned}
& g_{1, \mathrm{DECam}}=0.102 \pm 0.026 \\
& r_{1, \mathrm{DECam}}=0.113 \pm 0.021 .
\end{aligned}
$$

These values are consistent within the uncertainties with the zero-points derived by the DECam SMASH survey of the Magellanic Clouds (Nidever et al. 2017).

Unfortunately, we were not able to derive an airmass term from our data set, and therefore we used the same zero-points for all the DECam data we processed. If we assume that the missing airmass term is similar to those derived for Clay and CFHT, the uncertainty introduced in the zero-points by not correcting for this effect is of the order of $0.05 \mathrm{mag}$ in the $g$-band and $0.02 \mathrm{mag}$ in the $r$-band. We include these values when estimating the global photometric uncertainties and the subsequent luminosity values derived from them.

For an additional six satellites, Laevens 1 (Laevens et al. 2014), also known as Crater (Belokurov et al. 2014), Triangulum II (Laevens et al. 2015b), Horologium I I (Kim \& Jerjen 2015b), Hydra II (Martin et al. 2015), Kim 1 (Kim \& Jerjen 2015a) and Kim 2 (Kim et al. 2015b), and Balbinot 1 (Balbinot et al. 2013), the respective authors were kind enough to send us their photometric catalogs for the purpose of measuring their structural parameters.

\section{Consistency Checks}

\subsection{Comparison of CFHT and Clay Photometry}

Three objects in our primary sample were observed using both CFHT and Clay: Palomar 3, Segue 1, and NGC 7492. This provides us with an opportunity to assess the overall homogeneity of the photometry obtained with the northern and southern facilities. Although the exposure times 

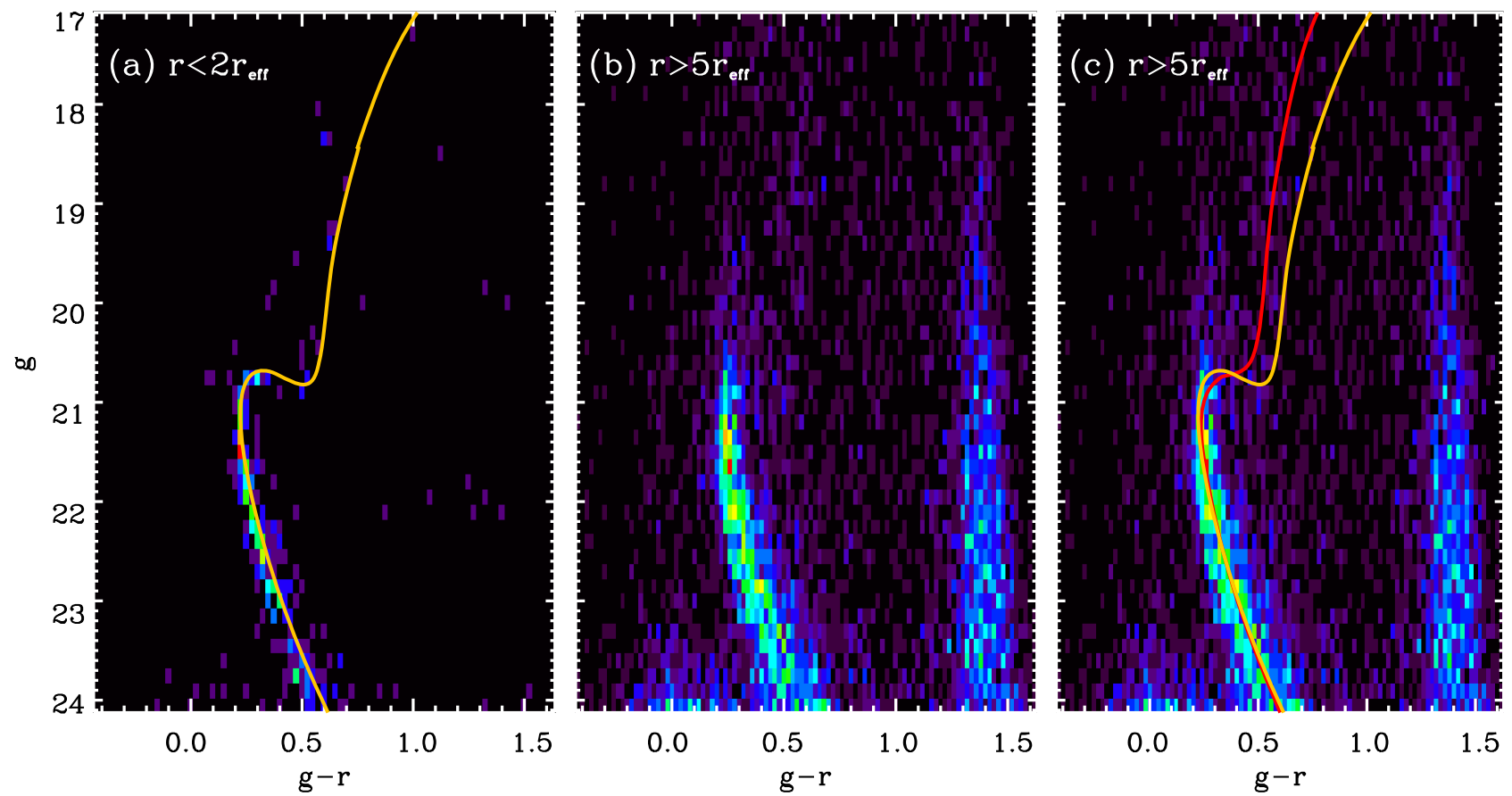

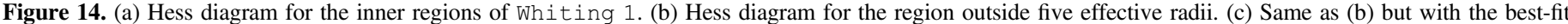
isochrone overlaid, indicating that the secondary main sequence is a similar distance as the cluster.
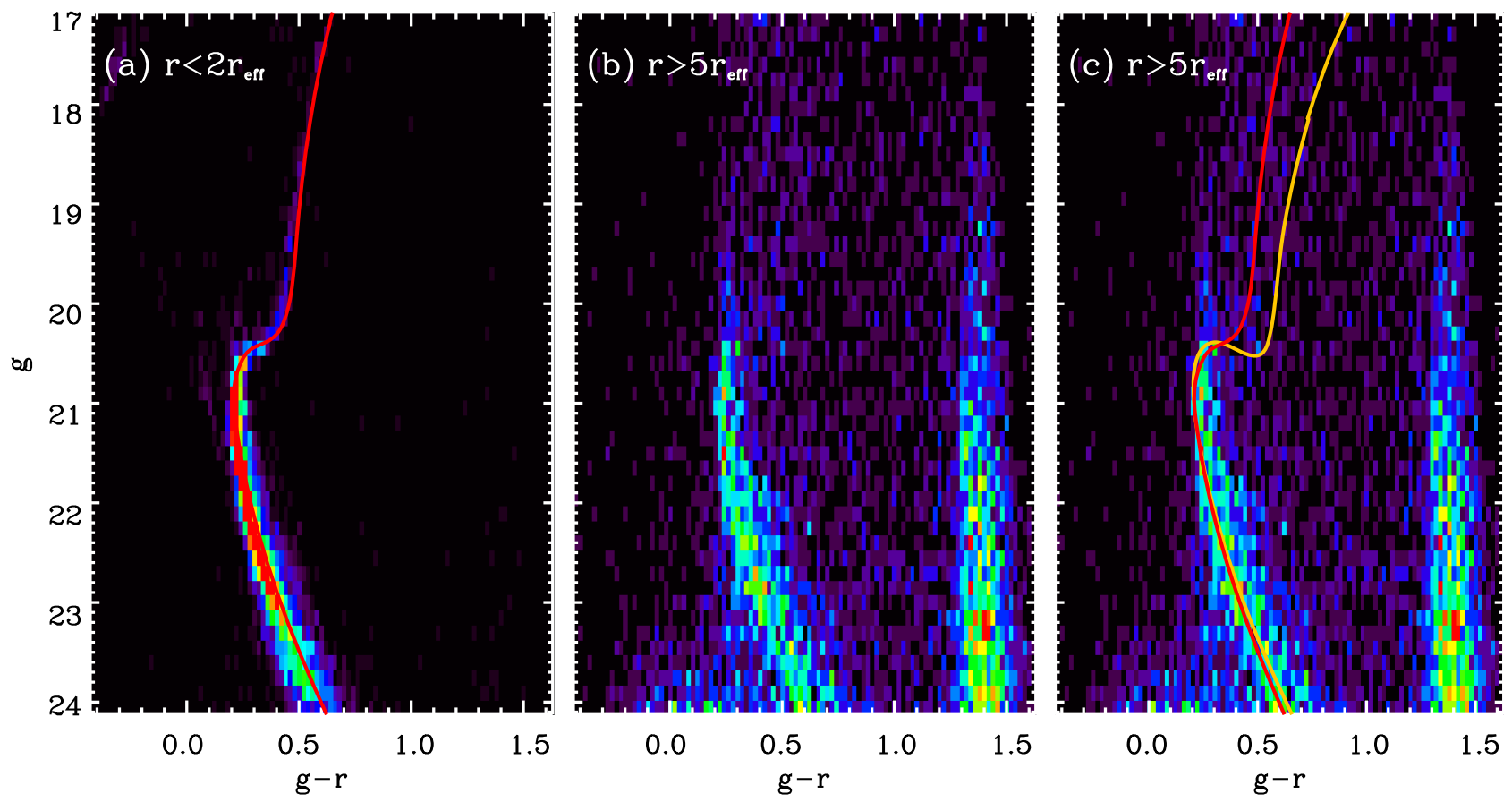

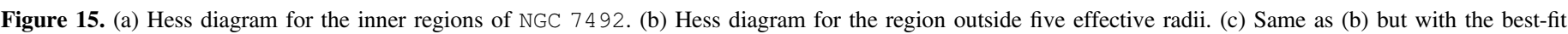
isochrone overlaid, indicating that the secondary main sequence is at a similar distance as the cluster.

were slightly shorter for our Clay observations (see Table 4 for details), this is roughly offset by the larger telescope aperture, resulting in comparable depths in both objects. Because a single pointing was used for both targets, though, the CFHT data have the advantage of covering a $\sim$ five times larger field.

The upper and lower panels of Figure 6 show CMDs for the inner regions of Palomar 3 and NGC 7492, respectively. Results from Clay are shown in the left panels, while those from CFHT are shown on the right. A visual inspection of this figure shows that the data are of comparable quality at the bright end, but the Clay-Megacam data are deeper, which is evident by the narrower main sequence at the faint end. To compare the photometry from the two instruments, Figure 7 shows histograms for $\left(g_{\mathrm{Clay}}-g_{\mathrm{CFHT}}\right)$ and $\left(r_{\mathrm{Clay}}-r_{\mathrm{CFHT}}\right)$ for both objects. This comparison uses sources down to $r_{\text {Clay }}=24$ and applies a cut of $-0.5<$ sharp $<0.5$ to isolate only star-like detections. For Palomar 3, the distributions are centered on $\left(g_{\text {Clay }}-g_{\text {CFHT }}\right)=0.011$ and $\left(r_{\text {Clay }}-r_{\text {CFHT }}\right)=0.022$ with dispersions of $\sim 0.031$ and $\sim 0.030$, respectively. In the case of NGC 7492, the distributions are centered on 


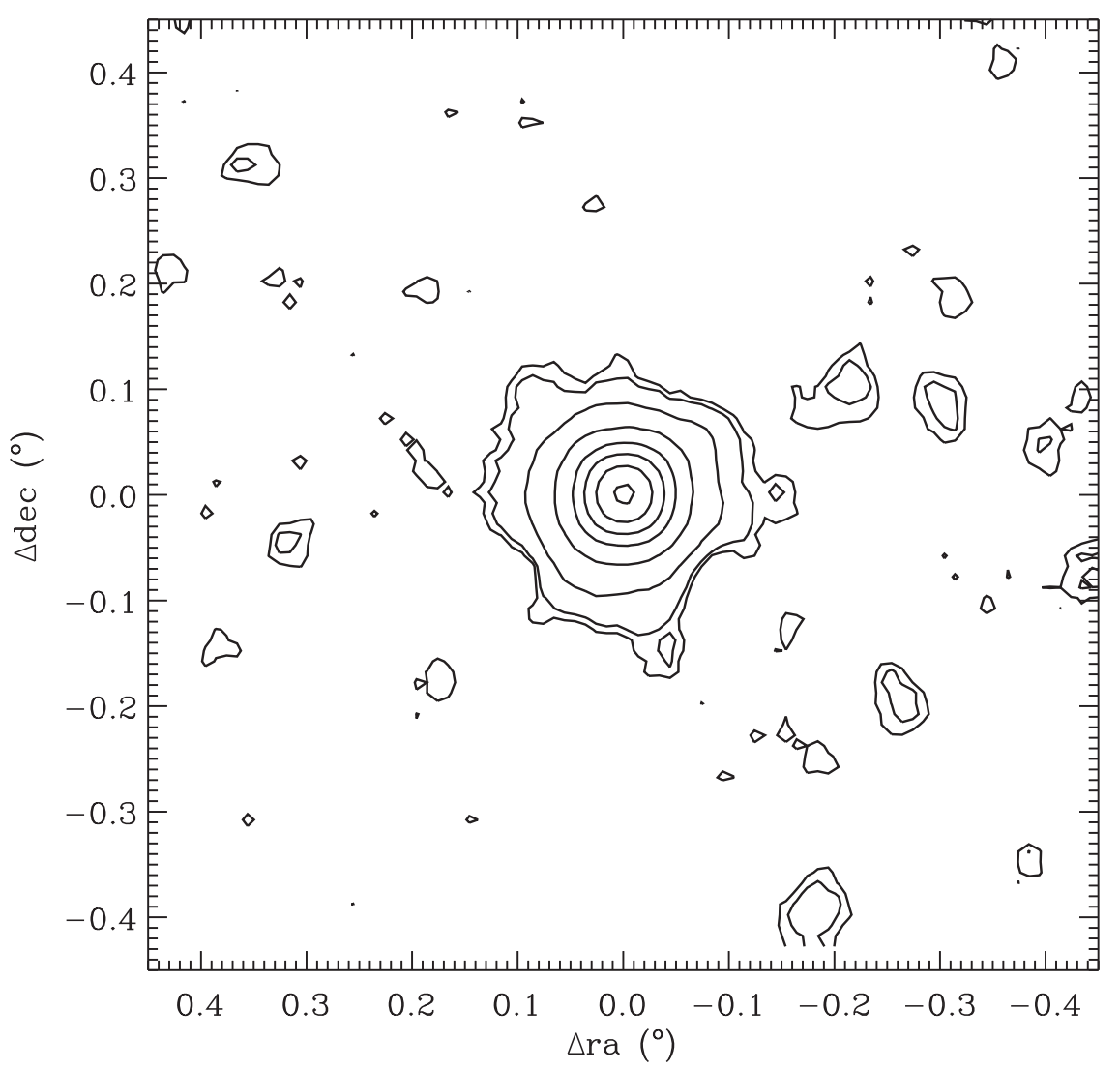

Figure 16. Density contour map for NGC 7492. The isodensity contours shown correspond to $2,3,10,50,200,450,850$, and $1450 \sigma$ over the background level.

$\left(g_{\text {Clay }}-g_{\text {CFHT }}\right)=0.038$ and $\left(r_{\text {Clay }}-r_{\text {CFHT }}\right)=0.049 . \quad$ The respective dispersions are 0.070 and 0.070 .

\subsection{Comparison of MegaCam and SDSS Photometry}

Deep, homogeneous photometry for our program objects is essential if we are to achieve the scientific goals laid out in Section 1: namely, the analysis of CMDs, star formation histories, density distributions, and structural parameters for a nearly complete sample of outer halo satellites. An obvious point of comparison is the SDSS, which has had an enormous impact on our census and understanding of the halo and its substructures. Recall from Section 3.3 that SDSS photometry is available for 27 of our 44 primary survey targets.

In Figure 8, we compare our new CMDs to those from SDSS (DR12) for four of our program objects. From top to bottom, the panels in this figure show CMDs for NGC 2419, CVn I, UMa II, and ComBer. Results from SDSS are shown in the left panels, while those from our program (all based on CFHT data) are shown on the right. The comparison has been restricted to the inner regions of the targets (roughly within their respective effective radii, the actual radii are shown in the figure). In all cases, the SDSS data were limited to unresolved sources with photometric uncertainties lower than 0.25 mag in both the $g$ - and $r$-bands. Similarly, the CFHT data were restricted to detections having $-0.5<$ sharp $<0.5$ and chi $<3$ in order to eliminate as many extended sources as possible. Similar restrictions on the $g$ and $r$ photometric errors were also applied.

For all four objects, Figure 8 shows there is a dramatic improvement in depth and precision compared to SDSS. The
SDSS CMDs typically reach only to a depth of $g \sim 22-23$ which is adequate only to identify the red giant branch at the distances of NGC 2419 and CVn I. For UMa I and ComBer, it is just possible to identify their MSTOs. By contrast, all the major evolutionary sequences are easily identified and well defined in the panels on the right, as the CFHT-MegaCam photometry reaches several magnitudes below the MSTO.

Figures 9 and 10 show histograms of the difference between our $g$ - and $r$-band magnitudes for a region of 20 arcmin around four objects. The left panels show the difference between CFHT and SDSS data for CVn 1 and NGC 2419, while the right panels show the difference between Clay and SDSS data for Palomar 3 and Leo II. Figure 11 shows the difference in $g$-magnitudes as a function of depth. All histograms are centered around zero, as expected, and their dispersions are consistent with the photometric uncertainties, typically larger for the shallower SDSS data.

As noted in Section 3, exposure times were chosen to ensure that our photometry would reach several magnitudes below the MSTO point in each of our program objects, irrespective of distance. This was achieved for most objects, except Leo T, CVn I, and NGC 2419. Figure 12 illustrates the depths reached in our primary survey. Results for the $g$ - and $r$-bands are shown in the upper and lower panels, respectively. The panels on the left show the approximate $5 \sigma$ limits for point sources in our MegaCam targets; there is a distribution in limiting magnitude that broadly peaks between $\sim 25$ and $26 \mathrm{AB}$ mag. In the right panels, we combine these limiting magnitudes with distances for our targets to show the approximate depths we reach below the MSTO. As expected, we see broad distributions in both bands that peak $\sim 2-3$ mag below the MSTO. 
Finally, in Figure 13 we compare the equatorial coordinates of the stellar-like detections $(-0.5<$ sharp $<0.5)$ for two objects observed by both imagers, Segue 1 and Palomar 3. The upper panels show histograms of the difference, in arcsec, between the positions determined from the Clay and the GAIA data for the same stars. The lower panels show the same differences but this time between Clay and CFHT data, to check for internal consistency. We note that stellar-like objects down to our magnitude limit are included.

\section{Results}

As an illustration of serendipitous findings from our catalog, we highlight two results.

\subsection{Sagittarius Debris in the Fields of Whiting 1 and NGC 7492}

One of the advantages of having relatively extended spatial coverage of our Galactic satellites is the possibility of studying their outer structure. This is particularly true in the case of globular clusters observed with CFHT, for which we typically cover several times their half-light (or equivalently their effective) radii. For a number of these clusters, secondary main sequences are observed beyond the cluster's extent. In some cases, such as NGC 2419 and Kop 2, the extra main sequences lie in the background or foreground of the clusters (Carballo-Bello et al. 2015). In other cases, like the ones presented here, the sequences seem to be at the same distance of the clusters, indicating either the presence of an extended structure related to the cluster (i.e., tidal tails or extended halos) or revealing the existence of a stream within which the clusters lie.

Whiting 1 lies at $\sim 30 \mathrm{kpc}$ from the Sun and is one of the youngest Galactic globular clusters known to date with an estimated age of $~ 6.5 \mathrm{Gyr}$, according to Carraro et al. (2007). These authors also showed that Whiting 1's distance, position in the sky, and mean heliocentric radial velocity $\left(v_{h}=131 \mathrm{~km} \mathrm{~s}^{-1}\right)$ coincide almost exactly with the position and radial velocity of tidal debris from the Sgr dSph (Majewski et al. 2003; Law et al. 2005). They thus argue that the cluster's origin is most likely to be associated with this dwarf galaxy. Through the analysis of $N$-body simulations of the Sgr dSph+tail system, Law \& Majewski (2010) also associated Whiting 1 with Sgr. Figure 14 shows the Hess diagram for the region within two effective radii of the cluster (left panel) using our photometry. The cluster's main and scarcely populated evolved sequences are evident. Also shown in this panel is the best-fit Dartmouth isochrone (Dotter et al. 2008): a $6.5 \mathrm{Gyr},[\mathrm{Fe} / \mathrm{H}]=-0.7$ track located at $d_{\text {helio }}=30.5 \mathrm{kpc}$. The middle panel of this figure shows the same diagram but for a region beyond five effective radii, where the cluster stellar density is extremely low and thus the contribution of cluster stars should be negligible. A secondary main sequence is clearly visible in this region. Based on the results from Carraro et al. (2007) and Law \& Majewski (2010), we associate this "extra" population with debris from the Sgr galaxy.

According to Law et al.'s model, the Sgr population at the position of Whiting 1 should be a combination of both old trailing and younger leading-arm debris, where the age refers to the time when the stars became unbound to the galaxy. For reference, in the right panel of Figure 14 we overplot both a
$[\mathrm{Fe} / \mathrm{H}]=-1.4,12 \mathrm{Gyr}$ old, and $\mathrm{a}[\mathrm{Fe} / \mathrm{H}]=-0.5,6.5 \mathrm{Gyr}$ old isochrone located at distances of $d_{\text {hel }}=26$ and $30.5 \mathrm{kpc}$, respectively. These isochrones are meant to represent old and intermediate-age Sgr populations. The choice of age and metallicity is motivated by the metallicity and age distribution of Sgr stars along its tails, as reported by Chou et al. (2007), and the star formation history derived by Siegel et al. (2007). Given the lack of detectable stars in the sub- and red-giant branch regions of the CMD, with our current data we cannot discriminate between a young population located at a similar distance as the cluster and an old but slightly closer one. However, we consider it likely that this Sgr population is a combination of ages and metallicities, and that it spreads in distance over a range of a few kiloparsecs.

Another globular cluster located close to tidal debris from the Sgr dSph is NGC 7492. In this case, Law \& Majewski (2010) found a coincidence between the spatial position of the cluster and its distance with those of Sgr debris, but considered a connection between the cluster and the galaxy unlikely based on the difference in mean radial velocity between the two systems. Figure 15 is the equivalent of 14 but this time showing Hess diagrams for our data in the field of the NGC 7492. The left panel again shows the central region of the cluster with the best-fit Dartmouth isochrones, a $13 \mathrm{Gyr}$, $[\mathrm{Fe} / \mathrm{H}]=-1.7$ sequence located at $d_{\text {helio }}=24 \mathrm{kpc}$. The middle panel shows a diagram for the outer regions of the cluster. Here, again, a secondary main sequence is clearly visible. Finally, the right panel is the same as the middle one but with the same set of isochrones shown in the right panel of Figure 14(c) overplotted. The old isochrone is located at the same distance as the cluster, while the younger isochrone is placed at $d_{\text {helio }}=28 \mathrm{kpc}$.

In addition to the potential connection to $\mathrm{Sgr}$ debris, it has been reported that NGC 7492 shows signs that it has been affected by Galactic tides. Using deep photometry, Lee et al. (2004) reported an elongated stellar distribution along with the presence of extended material asymmetrically distributed around the cluster (see their Figure 9). Our data set is slightly deeper and covers a larger area and thus we use it to investigate the potential extended structure of the cluster. Unfortunately, our results do not corroborate those of Lee et al. (2004). Figure 16 shows a density contour map for NGC 7492 where no clear elongation or tidal extensions are visible. We find an almost circular stellar distribution past the effective radius of the cluster, and cannot confirm the existence of an extended, asymmetrical structure surrounding the system. We suggest that the presence of $\mathrm{Sgr}$ debris at a similar distance as NGC 7492 affects the selection of cluster member stars based on CMD filtering, making it difficult to reach firm conclusions on the extended structure of this object.

\section{Summary}

We have described a new, systematic imaging survey of satellites belonging to the outer halo of the Milky Way (i.e., $R_{\mathrm{GC}} \geqslant 25 \mathrm{kpc}$ ). In a point of departure from most previous studies, our sample selection has been made with no constraint on morphology or classification. Our primary sample is composed of 44 objects for which we have acquired deep, wide-field (0.16-4 $\left.\mathrm{deg}^{2}\right) \mathrm{gr}$ imaging with the MegaCam instruments on the $3.6 \mathrm{~m}$ CFHT and the $6.5 \mathrm{~m}$ Magellan/Clay telescopes. The point-source limiting magnitude for our MegaCam imaging is typically $2-3$ mag below the MSTO in 
these objects in both bands. Collectively, the survey covers an area of $52 \mathrm{deg}^{2}$.

This sample has been supplemented with published photometry, or photometry derived from archival imaging, for 14 objects discovered between 2010 and 2015. Our final sample of 58 objects represents roughly three-quarters of outer halo satellites known as of 2015 (and roughly three-quarters of the currently known satellites). Our photometric catalog has already been used in a series of papers on outer halo satellites, their constituent stars, and possible foreground substructures (Bradford et al. 2011; Muñoz et al. 2012a; Santana et al. 2013; Carballo-Bello et al. 2015, 2017). In a companion paper, we present homogeneous photometric and structural parameters for these satellites (Muñoz et al. 2018), and in future papers we will examine their scaling relations in the context of other stellar systems.

The authors thank Dongwon Kim, Helmut Jerjen, and Eduardo Balbinot for kindly sharing their photometric catalogs for several of our secondary targets. We also thank David Nidever for providing code used for the SMASH survey to help derive astrometric solutions using GAIA as reference and an anonymous referee for helping us improve this article. This work was supported in part by the facilities and staff of the Yale University Faculty of Arts and Sciences High Performance Computing Center. R.R.M. acknowledges partial support from project BASAL PFB-06, as well as FONDECYT project $\mathrm{N}^{\circ} 1170364$. M.G. acknowledges support from the National Science Foundation under award number AST0908752 and the Alfred P. Sloan Foundation. S.G.D. was supported in part by the NSF grants AST-1313422, AST1413600, AST-1518308, and by the Ajax Foundation. This work was supported in part by the facilities and staff of the Yale University Faculty of Arts and Sciences High Performance Computing Center.

\section{ORCID iDs}

Patrick Côté (iD https://orcid.org/0000-0003-1184-8114 Felipe A. Santana (iD https://orcid.org/0000-0002-4023-7649 Marla Geha (ib https://orcid.org/0000-0002-7007-9725 Grecco A. Oyarzún (iD https://orcid.org/0000-0003-0028-4130 Peter B. Stetson (10) https://orcid.org/0000-0001-6074-6830 S. G. Djorgovski (i) https://orcid.org/0000-0002-0603-3087

\section{References}

Abazajian, K. N., Adelman-McCarthy, J. K., Agueros, M. A., et al. 2009, ApJS, 182, 543

Abell, G. O. 1955, PASP, 67, 258

Arp, H., \& van den Bergh, S. 1960, PASP, 72, 48

Balbinot, E., Santiago, B. X., da Costa, L., et al. 2013, ApJ, 767, 101 Belokurov, V., Irwin, M. J., Koposov, S. E., et al. 2014, MNRAS, 441, 2124 Belokurov, V., Walker, M. G., Evans, N. W., et al. 2008, ApJL, 686, L83 Belokurov, V., Walker, M. G., Evans, N. W., et al. 2009, MNRAS, 397, 1748 Belokurov, V., Walker, M. G., Evans, N. W., et al. 2010, ApJL, 712, L103 Belokurov, V., Zucker, D. B., Evans, N. W., et al. 2006, ApJ, 647, L111 Belokurov, V., Zucker, D. B., Evans, N. W., et al. 2007, ApJ, 654, 897 Bertin, E. 2006, in ASP Conf. Ser. 351, Astronomical Data Analysis Software and Systems XV, ed. C. Gabriel et al. (San Francisco, CA: ASP), 112 Bertin, E., \& Arnouts, S. 1996, A\&AS, 117, 393 Boulade, O., Charlot, X., Abbon, P., et al. 2003, Proc. SPIE, 4841, 72 Bradford, J. D., Geha, M., Muñoz, R. R., et al. 2011, ApJ, 743, 167 Cannon, R. D., Hawarden, T. G., \& Tritton, S. B. 1977, MNRAS, 180, 81P Carballo-Bello, J. A., Corral-Santana, J. M., Martínez-Delgado, D., et al. 2017, MNRAS, 467, L91
Carballo-Bello, J. A., Muñoz, R. R., Carlin, J. L., et al. 2015, ApJ, 805, 51, (Paper IV)

Carollo, D., Beers, T. C., Chiba, M., et al. 2010, ApJ, 712, 692

Carollo, D., Beers, T. C., Lee, Y. S., et al. 2007, Natur, 450, 1020

Carraro, G, Zinn, R., \& MoniBidin, C. 2007, A\&A, 466, 181

Cesarsky, D. A., Lequeux, J., Laustsen, S., et al. 1977, A\&A, 61, L31

Chou, M., Majewski, S. R., Cunha, K., et al. 2007, AJ, 670, 346

Dehnen, W., McLaughlin, D. E., \& Sachania, J. 2006, MNRAS, 369, 1688

Diehl, H. T., Abbott, T. M. C., Annis, J., et al. 2014, Proc. SPIE, 9149, 91490V

Djorgovski, S. 1993, in ASP Conf. Ser. 50, Structure and Dynamics of Globular Clusters, ed. S. G. Djorgovski \& G. Meylan (San Francisco, CA: ASP), 373

Dotter, A., Chaboyer, B., Jevremovic, D., et al. 2008, ApJS, 178, 89

Dunlop, J. 1828, RSPT, 118, 113

Elson, R. A. W., Fall, S. M., \& Freeman, K. C. 1987, ApJ, 323, 54

Flaugher, B., Diehl, H. T., Honscheid, K., et al. 2015, AJ, 150, 150

Gaia collaboration, Brown, A. G. A., Vallenari, A., et al. 2016, A\&A, 595, A2 Harrington, R. G., \& Wilson, A. G. 1950, PASP, 62, 118

Harris, W. E. 1996, AJ, 112, 1487

Harris, W. E. 2010, arXiv:1012.3224

Herschel, W. 1786, RSPT, 76, 457

Herschel, W. 1789, RSPT, 79, 212

Herschel, W. 1802, RSPT, 92, 477

Homma, D., Chiba, M., Okamoto, S., et al. 2016, ApJ, 832, 21

Hubble, E. P. 1930, ApJ, 71, 231

Irwin, M., \& Hatzidimitriou, D. 1995, MNRAS, 277, 1354

Irwin, M. J., Belokurov, V., Evans, N. W., et al. 2007, ApJL, 656, L13

Irwin, M. J., Bunclark, P. S., Bridgeland, M. T., \& McMahon, R. G. 1990 MNRAS, 244, 16P

Kim, D., \& Jerjen, H. 2015a, ApJ, 799, 73

Kim, D., \& Jerjen, H. 2015b, ApJL, 808, L39

Kim, D., Jerjen, H., Mackey, D., Da Costa, G. S., \& Milone, A. P. 2015a, ApJL, 804, L44

Kim, D., Jerjen, H., Milone, A. P., Mackey, D., \& Da Costa, G. S. 2015b, ApJ, 803,63

King, I. 1962, AJ, 67, 471

King, I. R. 1966, AJ, 71, 276

Kirby, E. N., Simon, J. D., \& Cohen, J. G. 2015, ApJ, 810, 56

Klypin, A., Kravtsov, A. ,V., Valenzuela, O., \& Prada, F. 1999, ApJ, 522, 82

Klypin, A., Zhao, H., \& Somerville, R. S. 2002, ApJ, 573, 597

Koposov, S., de Jong, J. T. A., Belokurov, V., et al. 2007, ApJ, 669, 337

Koposov, S. E., Belokurov, V., Torrealba, G., \& Evans, N. W. 2015a, ApJ, 805,130

Koposov, S. E., Casey, A. R., Belokurov, V., et al. 2015b, ApJ, 811, 62 Laevens, B. P. M., Martin, N. F., Bernard, E. J., et al. 2015a, ApJ, 813, 44 Laevens, B. P. M., Martin, N. F., Ibata, R. A., et al. 2015b, ApJL, 802, L18 Laevens, B. P. M., Martin, N. F., Sesar, B., et al. 2014, ApJL, 786, L3 Lauberts, A. 1976, A\&A, 52, 309

Law, D. R., Johnston, K. V., \& Majewski, S. R. 2005, ApJ, 619, 807 Law, D. R., \& Majewski, S. R. 2010, ApJ, 718, 1128

Lee, K. H., Lee, H. M., Fahlman, G. G., et al. 2004, AJ, 128, 2838 Luque, E., Pieres, A., Santiago, B., et al. 2017, MNRAS, 468, 97

Luque, E., Queiroz, A., Santiago, B., et al. 2016, MNRAS, 458, 603

Madore, B. F., \& Arp, H. C. 1982, PASP, 94, 40

Majewski, S. R., Skrutskie, M. F., Weinberg, M. D., et al. 2003, ApJ, 599, 1082

Martin, N. F., Nidever, D. L., Besla, G., et al. 2015, ApJL, 804, L5

Mateo, M. 1998, ARA\&A, 36, 435

McConnachie, A. W. 2012, AJ, 144, 4

McLaughlin, D. E., \& van der Marel, R. P. 2005, ApJS, 161, 304

McLeod, B., Geary, J., Conroy, M., et al. 2015, PASP, 127, 366

Moore, B., Ghigna, S., Governato, F., et al. 1999, ApJ, 524, L19

Muñoz, R. R., Côté, P., Santana, F. A., et al. 2018, ApJ, 860, 66

Muñoz, R. R., Geha, M., Côté, P., et al. 2012a, ApJ, 753, L15

Muñoz, R. R., Geha, M., \& Willman, B. 2010, AJ, 140, 138

Muñoz, R. R., Padmanabhan, N., \& Geha, M. 2012b, ApJ, 745, 127

Nidever, D. L., Olsen, K., walker, A. R., et al. 2017, AJ, 154, 199

Plummer, H. C. 1911, MNRAS, 71, 460

Pryor, C., \& Meylan, G. 1993, in ASP Conf. Ser. 50, Structure and Dynamics of Globular Clusters, ed. S. G. Djorgovski \& G. Meylan (San Francisco, CA: ASP), 357

Sakamoto, T., \& Hasegawa, T. 2006, ApJL, 653, L29

Santana, F. A., Muñoz, R. R., Geha, M., et al. 2013, ApJ, 774, 106, (Paper II) Schlafly, E. F., \& Finkbeiner, D. P. 2011, ApJ, 737, 103

Sérsic, J. L. 1968, Atlas de Galaxias Australes (Cordoba: Observatorio Astronomico) 
Shapley, H. 1938a, BHarO, 908, 1

Shapley, H. 1938b, Natur, 142, 715

Siegel, M. H., Dotter, A., Majewski, S. R., et al. 2007, ApJ, 667, L57

Simon, J. D., Drlica-Wagner, A., Li, T. S., et al. 2015, ApJ, 808, 95

Stetson, P. B. 1993, in IAU Coll. 136, Stellar Photometry, Current Techniques and Future Developments, ed. C. J. Butler \& I. Elliot (Cambridge: Cambridge Univ. Press), 291

Stetson, P. B. 1994, PASP, 106, 250

The DES Collaboration, Bechtol, K., Drlica-Wagner, A., et al. 2015, ApJ, 807,50

Torrealba, G., Koposov, S. E., Belokurov, V., et al. 2016a, MNRAS, 463, 712

Torrealba, G., Koposov, S. E., Belokurov, V., \& Irwin, M. 2016b, MNRAS, 459,2370

Trager, S. C., King, I. R., \& Djorgovski, S. 1995, AJ, 109, 218
Walsh, S. M., Jerjen, H., \& Willman, B. 2007, ApJL, 662, L83

Weinberger, R. 1995, PASP, 107, 58

Whiting, A. B., Hau, G. K. T., \& Irwin, M. 2002, ApJS, 141, 123

Willman, B., Blanton, M. R., West, A. A., et al. 2005a, AJ, 129, 2692

Willman, B., Dalcanton, J. J., Martinez-Delgado, D., et al. 2005b, ApJL, 626, L85

Wilson, A. G. 1955, PASP, 67, 27

Wilson, C. P. 1975 , AJ, 80, 175

Xue, X. X., Rix, H. W., Zhao, G., et al. 2008, ApJ, 684, 1143

York, D. G., Adelman, J., Anderson, J. E., Jr., et al. 2000, AJ, 120, 1579

Zucker, D. B., Belokurov, V., Evans, N. W., et al. 2006a, ApJL, 650, L41

Zucker, D. B., Belokurov, V., Evans, N. W., et al. 2006b, ApJL, 643, L103

Zwicky, F. 1959, Carnegie Inst. Washington Yearbook, 58, 60 